\title{
Graphene toxicity as a double-edged sword of risks and exploitable opportunities: A critical analysis of the most recent trends and developments
}

Yuri Volkov

Trinity College Dublin, Ireland, yvolkov@tcd.ie

Jennifer McIntyre

Technological University Dublin, jennifer.mcintyre@tudublin.ie

Adriele Prina-Mello

Trinity College Dublin, Ireland, prinamea@tcd.ie

Follow this and additional works at: https://arrow.tudublin.ie/nanolart

Part of the Chemistry Commons, and the Nanotechnology Commons

\section{Recommended Citation}

Volkov, Yuri \& McIntyre, Jennifer \& Prina-Mello, Adriele. (2016). Graphene toxicity as a double-edged sword of risks and exploitable opportunities: A critical analysis of the most recent trends and developments. 2D Materials. 4. 10.1088/2053-1583/aa5476.

This Article is brought to you for free and open access by the NanoLab at ARROW@TU Dublin. It has been accepted for inclusion in Articles by an authorized administrator of ARROW@TU Dublin. For more information, please contact arrow.admin@tudublin.ie, aisling.coyne@tudublin.ie,gerard.connolly@tudublin.ie.

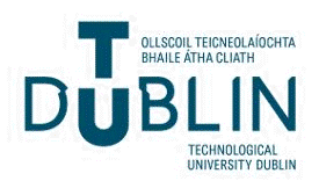


TOPICAL REVIEW

Graphene toxicity as a double-edged sword of risks and exploitable opportunities: a critical analysis of the most recent trends and developments

To cite this article: Yuri Volkov et al 2017 2D Mater. 4022001
You may also like

Nanotoxicity prediction using computational modelling - review and future directions

Bhavna Saini and Sumit Srivastava

Prenatal low-dose effects of pesticides and their long-term effects

L K Gerunova, E G Bardina and I V

Sechkina

- Toxicity of superparamagnetic iron oxide nanoparticles: Research strategies and

implications for nanomedicine

Lei Li, , Ling-Ling Jiang et al.

View the article online for updates and enhancements. 


\title{
2D Materials
}

\section{TOPICAL REVIEW}

\section{Graphene toxicity as a double-edged sword of risks and exploitable opportunities: a critical analysis of the most recent trends and developments}

RECEIVED
2 September 2016 REVISED

30 November 2016 ACCEPTED FOR PUBLICATION 13 December 2016

PUBLISHED

25 January 2017

\author{
Yuri Volkov ${ }^{1,3}$, Jennifer McIntyre ${ }^{2}$ and Adriele Prina-Mello \\ 1 School of Medicine and AMBER Centre, Trinity College Dublin, the University of Dublin, Dublin 2, Ireland \\ 2 Nanolab Research Centre, FOCAS Institute, Dublin Institute of Technology, Kevin Street, Dublin 8, Ireland \\ 3 Author to whom any correspondence should be addressed. \\ E-mail:yvolkov@tcd.ie
}

Keywords: graphene, toxicology, risk assessment, theranostics, health and safety, biocompatibility, nanomaterials

\begin{abstract}
Increased production volumes and a broadening application spectrum of graphene have raised concerns about its potential adverse effects on human health. Numerous reports demonstrate that graphene irrespective of its particular form exerts its effects on a widest range of living organisms, including prokaryotic bacteria and viruses, plants, micro- and macro-invertebrates, mammalian and human cells and whole animals in vivo. However, the available experimental data is frequently a matter of significant divergence and even controversy. Therefore, we provide here a critical analysis of the most recent (2015-2016) reports accumulated in the graphene-related materials biocompatibility and toxicology field in order to elucidate the cutting edge achievements, emerging trends and future opportunities in the area. Experimental findings from the diverse in vitro and in vivo model systems are analysed in the context of the most likely graphene exposure scenarios, such as respiratory inhalation, ingestion route, parenteral administration and topical exposure through the skin. Key factors influencing the toxicity of graphene and its complex derivatives as well as potential risk mitigation approaches exploiting graphene physicochemical properties, surface modifications and possible degradation pathways are also discussed along with its emerging applications for healthcare, diagnostics and innovative therapeutic approaches.
\end{abstract}

\section{Introduction}

From the utmost importance point of human health and safety perspective, the continuously expanding range of applications of carbonaceous nanomaterials dictates a necessity for the strict assessment of the associated hazards, potential risks in the context of the most likely exposure scenarios and the investigation of possible risk mitigation measures. Graphene in particular, being at the forefront of $2 \mathrm{D}$ representatives since the announcement of a Nobel Prize in the area in 2010, deserves a special level of merit and scrutiny alike.

The field of graphene toxicity has been previously overviewed in a number of publications addressing the complex issues of the synthetic routes [1], nanointerplay of biological objects and graphene as a function of its physiochemical properties [2], environmental exposure [3], tissue engineering [4], reproductive and developmental toxicity [5], biosensors and bioimaging applications [6-8], new nanodrug deliv- ery systems, advanced treatment and theranostic tools development [7, 9-14].

However, very recently accumulated experimental evidence demonstrates that there is still a considerable amount of uncertainty and sometimes controversy over the current findings related to the biocompatibility, toxicity and potential applications of graphene-based nanomaterials. This partially owes to the discrepancies in the experimental setups and approaches at different research centres, and to a significant extent also to the diversity of graphene forms available at the moment. Graphene is a single-layer 2D carbon nanomaterial that is made up of $s p^{2}$ hexagonal networks where strong covalent bonds are formed between adjacent carbon atoms [15]. 3D graphite is made up of layers of these hexagonal networks whereas rolled up sheets form $2 \mathrm{D}$ carbon nanotubes [16]. Graphene has many different forms better known as the graphene family nanomaterials, such as graphene oxide, reduced graphene oxide, single or few layer graphene [17], nanoribbons [18, 19], 


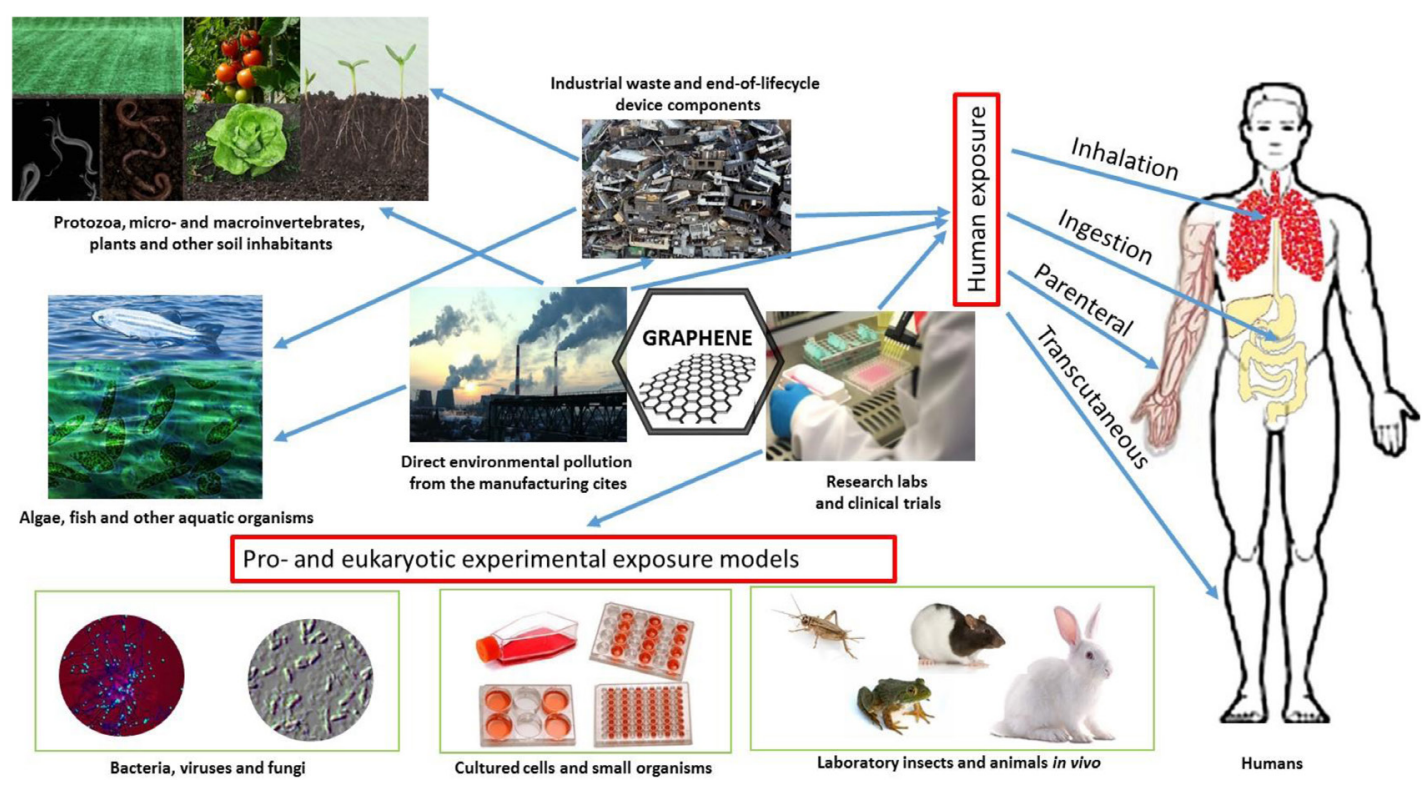

Figure 1. Main sources, exposure routes of graphene to living organisms and their experimental equivalents, and the ecosystems inhabitants subject to most significant exposure risk. The figure is an original illustration by the authors.

nanosheets, nanoonions [20] and graphene quantum dots $[19,21]$. All of them have very different edge effects and surface chemistries, for example graphene has a hydrophilic surface allowing for biointeractions predominantly at the edges or defect sites whereas graphene oxide is hydrophilic due to the many oxygen containing functional groups on the basal plane and edges, thus allowing for stable dispersions in water [22]

The awareness of the potentially negative consequences of graphene exposure to living organisms in general has been previously raised in this journal [23]. Therefore, we have aimed here to provide a more comprehensive overview of the most recent developments in the graphene-related biocompatibility and toxicology field predominantly accumulated over the past couple of years in order to elucidate the cutting edge trends and future opportunities in the area. Graphene and its derivatives are being increasingly exploited in advanced structures and devices designed to be used in electronics, catalysis, ICT and healthcare. In this case, one must consider the potential adverse environmental and health impact of graphene materials when released from the devices at the end of their lifecycle, largely focussing on the evaluation of potential hazards associated with the individual device components and implemented materials. On the other hand, a number of innovative systems for drug delivery which assume an immediate contact with human cells upon topical or systemic administration has been also suggested. Therefore, in order to get a comprehensive picture of the emerging 'nano-risks' fully applicable to graphene and graphenederived nanomaterials, devices and complex systems such as those intended for drug delivery, a multi-step approach must be implemented including hazard identification, characterisation and assessment of the most likely exposure scenarios. The identification and characterisation of the potential hazards is expected to involve a maximally detailed physicochemical characterisation of the nanomaterial under question and to include such parameters as size, shape, geometry and surface properties, aspect ratio, charge, water solubility, aggregation state, chemical composition including the potential contaminants during the synthetic process etc. In the industrial settings, one must also take into account such factors as the nanomaterial production volumes, material flow and potential particle release routes into the environment. Exposure assessment shall address a whole spectrum of living biological systems, including bacteria and other microorganisms, in vitro cultured primary cells and established cell lines, invertebrates and primitive multicellular organisms, lower vertebrates and in vivo in experimental animals implementing scenarios and models most closely imitating human contact with nanomaterials in real life. Here we provide the examples of such assessment in a wide range of living organisms.

The most common exposure routes of humans to nanomaterials are via inhalation, gastrointestinal tract after ingestion, topical exposure through the skin and parenteral or intravenous route, especially by intentional systemic administration. Both acute and chronic long-term exposure effects along with the nanomaterial bio-persistence and accumulation patterns must be thoroughly investigated in order to gain a most complete toxicological information. We analyse the most recently accumulated reports on graphene toxicity in the context of the above mentioned factors (figure 1). The possibilities of relevant risk reduction and management are evaluated including the opportunities to exploit graphene physicochemical properties, surface modifications and possible catabolic degradation pathways. Finally we provide a critical assessment of the new emerging applications of graphene-enabled products for healthcare, diagnostics and innovative therapeutic approaches. 


\section{Graphene effects on microorganisms, protozoa, plants, invertebrates and lower vertebrates}

\subsection{Bacteria and viruses}

Among the earlier reports addressing the influence of fundamental physical characteristics of graphene materials on microorganisms is the study by Liu et al [24] who have revealed the dependence of antibacterial activity of graphene oxide sheets on their lateral dimension. In this study carried out in the Escherichia coli model the authors explored the properties of graphene oxide sheets with over 100 times lateral size difference. As a result, it was established that larger graphene oxide sheets possessed a higher antibacterial activity than the smaller ones in a time- and concentration-dependent manner. As this phenomenon was not related either to the graphene sheets aggregation state or their oxidation capacity, it has been suggested that the bactericidal effect is mostly due to the specifics of direct interactions of graphene oxide particles of different size with bacterial cell walls, whereby larger graphene sheets cover the bacteria preventing the proliferation, whilst the similar in nature but smaller nanomaterials just adhere to the surface of bacteria and do not provide an efficient isolation of cells from the environment. A more recent work by Perreault et al [25] conducted in the same E. coli model microorganism system, graphene oxide sheets ranging between 0.01 and $0.65 \mu \mathrm{m}^{2}$ have been investigated for their antibacterial activity. They have established that surface coating using the smaller $0.01 \mu \mathrm{m}^{2}$ size nanosheets possessed a four times higher antimicrobial effect than using $0.65 \mu \mathrm{m}^{2}$ graphene oxide flakes, due to the higher defect density of smaller size graphene particles. Interestingly, this size-dependent effect of graphene oxide sheets on bacterial viability was reversed when the nanomaterials were used with cell suspensions, apparently due to the more efficient bacterial entrapment provided by larger graphene sheets.

Similar fundamental physical characteristics such as sharp edges and overall negative charge seem to be involved in broad spectrum antiviral activity of graphene oxide in pristine and reduced forms against pseudorabies DNA virus and porcine epidemic diarrhoea RNA virus. Moreover, graphene oxide was capable of causing a structural damage to both virus types prior to cell entry [26].

An interesting comparative toxicological analysis study of different graphene material types has been conducted by Efremova et al [27]. They have investigated the original graphene shells and their derivatives graphene oxide and graphene oxide paper in luminescent E. coli reporter system addressing the nanomaterials' toxicity and bioactivity. Of these, only graphene oxide has shown a marked toxicity however not related to the oxidative stress, but rather through the charge neutralisation, energy and transport pathways disruption in bacteria, therefore suggesting the direct contact membrane stress and graphene oxide internalisation as the leading mechanisms of toxicity. This report highlights the possibility of existence of graphene-related toxicity mechanisms operating either apart or in parallel to those included in the established paradigm of reactive oxygen species-dependent cell damage by nanomaterials, vindicating further studies in this direction.

However, in stark contrast to the data above, Barbolina et al demonstrated that graphene oxide in a highly purified form did not exert any bactericidal or growth-enhancing effects in several strains of $E$. coli and Staphylococcus aureus [28] at the concentrations as high as $1 \mathrm{mg} \mathrm{ml}^{-1}$, unlike the insufficiently washed graphene oxide samples which retained antimicrobial activity. The authors attribute this phenomenon to the impact of the acidic impurities on the nanomaterial surface which can be eliminated via implementing a strict purification protocol.

This fact once again stresses the importance of a thorough physico-chemical assessment of the prospective nano-enabled products at each stage of their development, to avoid the misleading results concealing the true activity spectrum of novel agents independent of their future specific applications. On this note, among the other most common contaminants which might contribute to rendering false positive efficacy readouts are endotoxins and the presence of these must be subject to scrutiny in any study addressing biological effects of the engineered nanomaterials, including the graphene-based [29].

Graphene nanomaterials exert their antibacterial properties not only on a wide range of pathogenic microorganisms, but also on the bacteria present in the environmental ecological systems. For instance, graphene oxide has been shown to inhibit growth and viability of Pseudomonas putida species, a common bacteria involved in wastewater treatment process. $P$. putida displayed a concentration-dependent decrease of activity evaluated by its ability to remove the pollutant salicylic acid from simulated urban and industrial wastewaters upon exposure to graphene [30]. Similar and long term effects of natural and industrial carbonaceous nanomaterials, including biochar, carbon black, multi-walled carbon nanotubes and graphene have been registered on the bacterial and fungal communities of soil, judged by the analysis of soil biomass accumulation and substrate-induced respiration [31]. Dose-dependent toxicity of synthesised graphene oxide has been also recently reported against methylotrophic yeast Pichia pastoris as a model industrial microorganism. In this case, key mechanisms suggested to be contributing in microbicide activity involved generation of reactive oxygen species and cell membrane damage. Upregulation of genes responsible for synthesis of unsaturated fatty acids has been identified in this study as a key protective mechanism enabling the cells to resist the damaging action of graphene oxide [32]. However, the database of the toxic effects of graphene and 
derivatives on the diverse bacterial representatives of the ecosystems is fragmentary at present and requires further significant efforts from the perspective of increased environmental exposure to the grapheneenabled products at the end of their lifecycle.

\subsection{Protozoa, algae and plants}

The studies disclosing potential toxic effects of graphene materials on this category of living organisms are of a prime importance from the eco-toxicological perspective, as the consequences of the exposure of the most abundant biomass- and oxygen- producers to damaging impacts can have very far-reaching global consequences.

Euglena gracilis as one of the typical aquatic protozoan organisms has been used by $\mathrm{Hu}$ et al [33] as a test model of exposure to graphene oxide ranging from $0.5 \mathrm{mg} \mathrm{l}^{-1}$ to $5 \mathrm{mg} \mathrm{l}^{-1}$ over $10 \mathrm{~d}$. The authors found that that the EC50 of graphene oxide after a $96 \mathrm{~h} \mathrm{long}$ incubation was $3.76 \pm 0.74 \mathrm{mgl}^{-1}$ and further concentration increase up to at $2.5 \mathrm{mg} \mathrm{l}^{-1}$ shown pronounced and significant damaging effects on the protozoa, as detected by growth inhibition, enhancement of malondialdehyde content and antioxidant enzyme activities. It has been suggested that shading effect and oxidative stress could be the leading factors responsible for observed graphene oxide toxicity. Similarly, graphene oxide nanosheets and graphene oxide quantum dots are able to reduce viability of another primitive freshwater organism Chlorella vulgaris. In this study it has been shown that graphene oxide nanosheets entrap the algae thereby reducing cell permeability, whereas much smaller graphene oxide quantum dots induced the shrinkage of the plasma membrane and enhanced cell permeability with facilitated internalization effects, plasmolysis and oxidative stress activation, leading to the inhibition of cell proliferation and chlorophyll biosynthesis [34]. The results of this study clearly highlight the size dependence of graphene toxicity in lower plant species. A different species of green algae-Raphidocelis subcapitata were also prone to toxic effect of graphene oxide starting from the concentrations as low as $10 \mu \mathrm{g} \mathrm{m} \mathrm{l}^{-1}$ [35].

In contrast to these data, it has been found that graphene oxide can in fact, exert a protective effect against copper-induced toxicity in a primitive freshwater plant organism microalga Scenedesmus obliquus at the environmentally relevant concentration of $1 \mathrm{mgl}^{-1}$ following a $12 \mathrm{~d}$ exposure, justifying the need for further studies on graphene-induced effects in ecologically significant organisms of different species [36].

From a very scarce number of original reports dedicated recently to graphene toxicity in higher plants, one can note a comparative study carried out on a selection of plant species including Lolium multiflorum (ryegrass), Solanum lycopersicum (tomato), and Lactuca sativa (lettuce) addressing the toxicity of the soluble graphitic nanofibers, multiwalled carbon nanotubes and graphene oxide. The latter has been found to be most toxic across the whole concentrations range, followed by graphitic nanofibres and carbon nanotubes which were deemed relatively non-toxic [37]. In agreement with the above, it has been demonstrated that graphene can also suppress the morphogenesis of rice seedlings in a concentration-dependent manner [38] and potentiate arsenic-induced phytotoxicity in wheat [39]. Interestingly, in the above mentioned study [38], the lowest concentration of graphene oxide of $5 \mathrm{mg}^{-1}$ controversially increased adventitious root number, fresh weight of root and over ground part of the seedlings. The mechanism of this phenomenon has not been disclosed and requires further investigation.

\subsection{Invertebrates and lower vertebrates}

A limited number of studies reported in the literature is dedicated to the investigation of graphene effects in a common macroinvertebrate Caenorhabditis elegans as an alternative in vivo toxicity testing model [40]. Multi-endpoint, high-throughput study of toxicity carried out using a panel of nanomaterials in C. elegans has been conducted by Jung et al [41] demonstrating that graphene was among the carbonaceous materials clearly exerting toxic effect on this organism, depending on the graphene oxidation state, with the graphene oxide being most toxic, flowed by reduced and pristine graphene. Notably, recent experiments carried out in this model enabled to reach a new level of understanding of the graphene-triggered toxicity and counteracting protective mechanisms. Thus, in the extensive genome-wide screening investigation with quantitative analysis, 34 dysregulated long noncoding RNAs with a potential for involvement in regulation of various biological processes have been identified as a result of exposure of this organism to graphene oxide. The authors identified the shared long noncoding RNAs, linc-37 and linc-14, involved in the control of chemical surface modifications and genetic mutations in alleviating graphene oxide toxicity. Furthermore, linc-37 binding to transcriptional factor FOXO/DAF-16 might be of key importance for the control of graphene oxide toxic impact [42]. In two reports complementing to these findings, Zhao et al used the same model to investigate effects of graphene oxide on C. elegans longevity and reproductive toxicity and suggested that the molecular mechanisms controlling graphene oxide toxicity possibly develop via insulin signalling pathway and through anti-apoptotic epigenetic signal encoded protection mechanism, respectively $[43,44]$.

Graphene oxide is not biologically inert either for the domestic crickets or for small aquatic vertebrates such as zebrafish. In Acheta domesticus cricket species, the authors studied in vivo toxicity of pure and manganese ions contaminated graphene oxide, which were injected into the haemolymph of the insect [45] over $1-72 \mathrm{~h}$ interval after the administration. The results pointed to the increased oxidative stress beyond $24 \mathrm{~h}$ after graphene injection, as reflected in the elevated activity of catalase and gluthiathione peroxidases and 
followed by raised heat shock protein HSP-70 levels. Exposure of zebrafish to graphene oxide revealed that at the concentration range of $1-50 \mathrm{mgl}^{-1}$ over two weeks there were no signs of acute toxicity despite the appearance of moderate histological changes in the liver and intestines and clear evidence of the oxidative stress reflected in elevated malondialdehyde levels, superoxide dismutase and catalase activities on the background of the reduced glutathione content in the liver. Notably, in this study Chen et al for the first time have also demonstrated that graphene oxide exposure induced immunotoxicity in this species, as evidenced by the raised of levels of TNF- $\alpha$, IL- $1 \beta$ and IL- 6 cytokines in the spleen [46]. The effects of much smaller graphene quantum dots were investigated in embryonic zebrafish at concentrations range from 12.5 to $200.0 \mu \mathrm{g} \mathrm{ml}^{-1}$ up and enabled to establish that the lowest graphene oxide concentration of $12.5 \mu \mathrm{g} \mathrm{ml}^{-1}$ was relatively non-toxic and even induced larval hyperactivity, whereas higher doses resulted in hypoactivity and further induced serous embryonic malformations at the concentrations exceeding $50 \mu \mathrm{g} \mathrm{ml}^{-1}$ [47].

The comparative toxicity of different forms of graphene such as pristine graphene, graphene oxide and reduced graphene oxide was investigated in a chicken embryo model at the concentrations of 50-5000 $\mu \mathrm{g} \mathrm{ml}^{-1}$. Interestingly, the survival rate of embryos decreased significantly following treatment with all types of graphene, but this phenomenon was not dosedependent. The body weights were only marginally affected by the highest doses of graphene, whereas the organ weights remained unaltered [48]. These findings could be better understood in the light of the work of Mottier et al carried out in a different model of lower vertebrate Xenopus laevis larvae, which clearly demonstrated that the surface area in contrast to mass concentration is the most accurate descriptor of toxicity induced by carbonaceous nanomaterials, including graphene [49].

Overall, despite the fact that the experiments in the invertebrates and lower vertebrates often help to unveil fundamental mechanisms (including nanotoxicological) operating in very similar ways in the higher animals, primates and humans and are indispensable for the development of relevant risk alleviation strategies, they deserve far more attention in relation to the emerging graphene materials than they received to date.

\section{In vitro mammalian cell and tissue models}

The studies addressing cytotoxicity of graphene materials in cultured mammalian cell models require a special attention in the context of this review due to several important considerations. Firstly, they have been and remain the most widely accepted in vitro model systems for the baseline safety screening and analysis of animal and human cell responses to a diverse range of potentially hazardous agents, including nanomaterials. Secondly, the majority of the cell lines have been isolated from the human samples and provide an excellent experimental panel for evaluating the performance of engineered prospective diagnostic tools and investigating the activity of new medicinal drugs and compounds for medical devices in a safeby-design manner. Lastly, the growing number of emerging in vitro experimental systems closely imitating physiological exposure scenarios enables to obtain valuable and precise information about the potential effects of innovative diagnostic, therapeutic and multifunctional (theranostic) tools in accordance with the requirements of the 3Rs (Replacement, Reduction and Refinement) approach to animal studies.

\subsection{Primary and established model cell cultures corresponding to major exposure routes}

The ubiquitous presence of phagocytic cells throughout the human body makes them a first line of defence against any type of foreign invasions independently of the exposure route; the engineered nanomaterials unfortunately are not constituting a lucky exception from the category of such invaders.

Therefore, in vitro macrophage cultures commonly provide a staple and universal exposure model system for evaluation of uptake, accumulation and cell functional responses assessment following the exposure to externally presented nanoparticles. Over the last couple of years, a growing amount of evidence has been accumulated in the literature demonstrating the ability of graphene oxide to induce pro-inflammatory responses in macrophages in vitro. Ma et al investigated the induction of such functional responses in phagocytes following the exposure to a panel of single-layered graphene oxide samples with differential lateral sizes derived from the identical starting material. They found that large (750-1300 nm) graphene oxide flakes showed a strong adsorption onto the plasma membrane and reduced phagocytosis, which facilitated closer interactions with toll-like receptors and a marked activation of NF- $k \beta$-mediated pathways. Smaller lateral size graphene oxide sheets were more actively taken up by cells. Consequently, larger graphene oxide particles promoted a stronger M1 type polarization, associated with enhanced production of inflammatory cytokines and recruitment of immune cells, as further confirmed in vivo by local and generalised inflammatory responses following graphene systemic administration. Hence, the size dependence of the phagocytic inflammatory response to graphene oxide has been clearly elucidated [50]. Another study conducted in RAW 264.7 macrophage cell line using carbon nanotubes, nanographite and carbon black revealed that these materials with similar physicochemical characteristics in pristine form or following acid treatment functionalisation in general induced a low level of cytotoxicity. Despite not exactly dealing with a single-layered graphene this report deserves attention here, since it has been convincingly shown that acid functionalization enhanced 


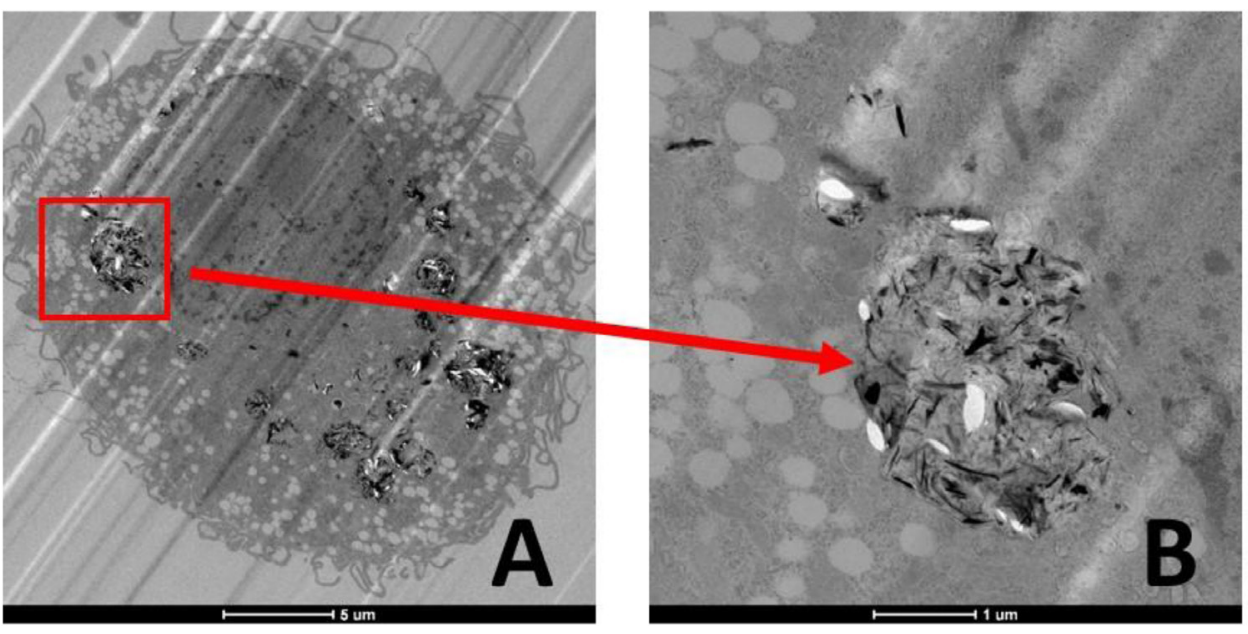

Figure 2. Transmission electron microscopy image of pristine graphene nanosheets uptake by primary human macrophage. (A) Graphene sheets are seen as electron-dense structures tightly packed within the lysosomal compartments of the cell. (B) High magnification zoom on the intracellular area marked by red rectangle in (A). The details of the experimental procedure are fully explained in [54] published by the authors of this review. Microphotographs correspond to the figure 5 of the publication, but were not selected for the original manuscript from the repeated consistent sets of experimental data and hence were not previously subject to copyright.

the ability of the diverse carbon materials to induce pro-inflammatory response, except for carbon black, as estimated by TNF-alpha production levels. Since at the equivalent surface chemistry and exposure the functional responses of macrophages to nanographite were higher than those for nanotubes and carbon black, the authors bring their data in support of the earlier suggested platelet and fibre paradigm [51]. Unidirectional pro-inflammatory effects have been registered by Wang et al [52] in a comparative study using multiwalled carbon nanotubes, graphene and graphene oxide which demonstrated the increased interleukinlbeta and transforming growth factor-betal secretion level in phagocytic and lung epithelial cell lines THP-1 and BEAS-2B upon exposure to these nanomaterials. Graphene oxide proved to be most potent in induction of pro-fibrogenic response type, in contrast to pluronic PF108-dispersed graphene and nanotubes, once again stressing importance of the dispersal state and surface reactivity on the ultimate outcome of the inflammatory process in the lungs.

More complex graphene oxide-silver nanocomposites along with their pristine precursors were used in a comparative toxicity study in murine phagocytic $\mathrm{J} 774$ cell line and peritoneal macrophages from Balb/c mice. It was found that the graphene oxide-silver nanocomposites were more toxic than the unmodified graphene oxide and silver nanoparticles in both types of macrophages under investigation, due to significantly higher reactive oxygen species production compared to pristine nanomaterials [53]. The results of our own recent studies [54] also show that pristine graphene is readily taken up by primary human macrophages without any significant acute functional or structural damage (figure 2), in contrast to pristine single walled carbon nanotubes triggering the autophagic type of cellular response.
A number of recent reports focussed on the toxic responses of human cells to graphene materials developing as a result of the potential specific exposure routes. Thus, primary human corneal epithelium cells (hCorECs) and human conjunctiva epithelium cells (hConECs) were implemented to investigate the dose-and time-dependent toxicity of graphene oxide in the case of occasional or repeated ocular exposure. Acute $2 \mathrm{~h}$ long graphene oxide exposure did not induce cytotoxicity to hCorECs in contrast to the short-term $24 \mathrm{~h}$ exposure causing a marked cytotoxicity both in hCorECs and hConECs with the raised indicators of oxidative stress in a dose-dependent manner between 12.5 and $100 \mu \mathrm{g} \mathrm{ml}^{-1}$. Of note, these findings correlate well with the results subsequently obtained in a rat and rabbit animal models [55].

A comparative study of cytotoxicity of graphene oxide and pristine graphene has been carried out in the model of skin fibroblasts which to some extent imitates the dermal exposure scenario. It established that the more compact graphene sheets proved to be more damaging to mammalian fibroblasts than the less densely packed graphene oxide [56].

The enteric ingestion route has been mimicked in the model of CaCo- 2 cells. Four different graphene oxide monolayer and aggregate samples were tested and despite the evidence of close interaction on nanomaterials with the cell surface and the formation of reactive oxygen species, no visible signs of acute toxicity were found for any of the graphene types up to $48 \mathrm{~h}$ exposure. Graphene nanoplatelets aggregates induced detectable, but low toxicity at higher concentrations up to $80 \mu \mathrm{g} \mathrm{ml}^{-1}$ strongly indicating that aggregation state and the number of graphene layers have a stronger impact on cytotoxicity than their lateral size [57].

In respect of the systemic parenteral exposure scenario, it is essential to characterise the consequences of 
the nanomaterials encounters with the key components of vascular system and blood. Graphene oxide has been shown to induce damaging effects on human red blood cells, as evidenced by the efflux of haemoglobin from erythrocytes in suspension. At the smallest particle size, graphene showed the strongest haemolytic activity, in contrast to the aggregated graphene sheets [56]. On the other hand, Chowdhury et al evaluated the interaction of more complex structures such as graphene nanoribbons with red blood cells, vascular endothelial cells, platelets, monocyte/macrophage cells, mast cells and complement proteins. Although the nanoribbons induced a low degree of concentration-dependent deformation of red blood cells, it did not lead to haemolysis. Exposure to the nanoribbons up to $80 \mu \mathrm{g} \mathrm{ml}^{-1}$ did not lead to any significant changes in the all the other components of the blood vascular system under study, except for endothelial cells which were actively taking up the graphene nanoribbons and exhibited a concentration dependent decrease in cell viability [18]. These data are in concert with the report confirming the damaging effect of few-layer graphene with lateral dimension of $\sim 160$ and thickness of $\sim 0.8 \mathrm{~nm}$ on DNA of human primary umbilical vein endothelial cells involving an organized oxidative stress paradigm [17]. Furthermore, graphene oxide has been also shown to exert a particular adverse effect on $\mathrm{B}$ cell functions and the humoral immunity, as confirmed by modulated B cell surface phenotype affecting CD80, CD86 and CD40 receptors and antigen presenting molecules. The secretion of immunoglobulins by terminally differentiated plasma cells was also markedly suppressed by graphene oxide [58].

Mimicking the inhalation exposure conditions in cell cultures imposes a significant level of complexity as the closest available model of air-liquid interface has been introduced quite recently on its own, and the data on graphene toxicity in this model are not yet available. In general, similar limitations imposed by the absence of reliable and validated models adequately imitating physiological 3D microenvironments presented by normal mammalian cells and interweaving connective tissue components currently apply to most experimental systems implementing non-transformed cell models and are likely to be improved in line with the future technological advances in cell and tissue culture approaches.

\subsection{Other normal mammalian cells and cell-free systems}

Among these publications, the impact of graphene nanostructures on the functional responses of mesenchymal stem cells (MSCs) deserves a special attention. Thus, Talukdar et al performed a comparative study of several 2D graphene nanostructures including graphene nano-onions, graphene oxide nanoribbons and graphene oxide nanoplatelets on viability and differentiation of human MSCs and arrived to the conclusion that despite active uptake, at the concentrations below $50 \mu \mathrm{g} \mathrm{ml}^{-1}$ they neither affected the cell function or MSCs differentiation potential into adipocytes, irrespective of the type of nanostructure used [20].

Recent literature in the field also for the first time includes a report on the cytotoxicity of graphene oxide and reduced graphene oxide on spermatogonial stem cells. This study showed that graphene oxide significantly increased oxidative stress at concentrations above $100 \mu \mathrm{g} \mathrm{ml}^{-1}$, leading to the reduction of the cell number. However, a reduced graphene treated sample in contrast, demonstrated a significant increase in cell proliferation [59].

Toxic effects of graphene oxides have been also investigated in several elegant physiologically relevant cell-free models. For example, it has been reported that graphene oxide nanosheets can affect the ultrastructure and biophysical properties of the pulmonary surfactant film, which sheds a new light on the inhalation toxicity potential of these materials. Upon deposition, the graphene oxide nanosheets induced pores in the pulmonary surfactant film thereby imposing adverse effects on its ultrastructure and biophysical properties. Notably, the pore formation induced by graphene nanosheets results in increasing the compressibility of the surfactant film and associated functional inhibition [60]. The propensity of graphene oxide to attach to and to disrupt model cell membranes has been investigated using supported lipid bilayers and supported vesicular layers composed of zwitterionic 1,2-dioleoyl-snglycero-3-phosphocholine. It was found that the attachment of graphene oxide particles to supported lipid bilayers was controlled by electrostatic interactions and the disruption of lipid vesicles. However, when the exposure of the supported vesicular layers to the nanoparticles was terminated, the pores on the lipid bilayers demonstrated a remarkable self-healing ability [61]. Potential cytotoxicity mechanism of graphene oxide was also investigated using large-scale, allatom molecular dynamics simulations to explore the mechanism of interactions between a HIV-1 integrase protein dimer and graphene nanosheets oxidized at different levels. The authors came to the conclusion that the graphene nanosheet could intercalate between the two monomers of the dimer, disrupting the proteinprotein interactions and eventually leading to dimer disassociation [62].

\section{In vivo animal models}

The reports addressing the issues of graphene toxicity in vivo in the laboratory animals can be analysed best from the angle of relevant exposure routes similarly to the above discussed in vitro systems, as this approach provides a more systematic information for the risk assessment process.

\subsection{Respiratory exposure route}

One of the most likely exposure scenarios potentially applicable both to the industrial manufacturing settings and for accidental environmental presentation 
of graphene to humans is the respiratory (inhalation) route.

Han et al [63] investigated effects of graphene oxide on the lungs of Sprague-Dawley rats in a single $6 \mathrm{~h}$ nasal inhalation technique at 0.46 and $3.76 \mathrm{mg} \mathrm{m}^{-3}$. They found that the exposure to graphene material did not cause any major changes in the body or organ weight and food consumption during the two weeks of recovery interval. Similarly, neither microalbumin nor lactate dehydrogenase content in the bronchoalveolar lavage fluid shown any significant changes. Same applied for total cell count and the number of macrophages, neutrophils and lymphocytes. Histologically, clear uptake of graphene oxide by the alveolar macrophages was detected only after exposure to the higher concentration. In a very close experimental system implementing nasal inhalation exposure in rats, but over a more lengthy period of $28 \mathrm{~d}$ and using the graphene oxide concentrations of $0.12,0.47$, and $1.88 \mathrm{mg} \mathrm{m}^{-3}$ followed by up to three months-long recovery, no gross body or organ mass changes were registered either. Despite the fact that the nanomaterial has been actively taken up by phagocytes and accumulated further in lymph nodes, no significant pathological alterations were observed in the lungs of the exposed rats [64]. Overall, these data witness a minimal level of graphene oxide pulmonary toxicity either after a single or multiple dose extended inhalation exposure even at the relatively high concentration which likely exceeds the potential realistic environmental equivalent.

In an alternative intratracheal instillation model, potential pro-inflammatory effects of graphene nanoplatelets and their accumulation in the mediastinal lymph nodes of rats has been addressed in the context of varying surface functionalisation of nanomaterial by $\mathrm{COOH}$ groups. All the tested graphene particles, whether in the original pristine form or decorated with $\mathrm{COOH}$ functional groups, demonstrated a high ability to induce generation of reactive oxygen species. In this work, both acute and chronic inflammogenic effects have been analysed after $24 \mathrm{~h}$ with the instilled doses of 0.3 and $1 \mathrm{mg} /$ rat and following 1 and 4 weeks at $3 \mathrm{mg} / \mathrm{rat}$ dose. Interestingly, only the positively charged graphene nanoplatelets demonstrated a significant increase of neutrophil counts after $24 \mathrm{~h}$, despite the fact that unidirectional dose-dependent effects were noticeable with all the particle types under study. The observed acute pro-inflammatory effect was not sustained and cell counts returned to normal already after 1 week. However, there was a progressive accumulation of nanomaterial in the regional lymph nodes over time, irrespective of its surface charge modification [65].

Pulmonary and systemic toxicity of different size graphite nanoplateles has been comparatively analysed by Roberts et al [66] in mice after pharyngeal aspiration followed at $4 \mathrm{~d}$ to 2 months post exposure. Despite the varying lateral $(2-20 \mu \mathrm{m})$ dimensions and thickness $(8-25 \mathrm{~nm})$ of nanoplatelet samples, at the dose of $4 \mu \mathrm{g}$ /animal no signs of toxicity were registered.
However, the exposure of mice to $40.0 \mu \mathrm{g}$ of graphite nanoplatelets with 5.0 and $20.0 \mu \mathrm{m}$ lateral dimensions lead to the pulmonary inflammation and altered tissue gene expression profile in the lungs, aorta and hepatic inflammatory and acute phase genes. Biodistribution and toxicity of radiolabelled graphene, following a similar intratracheal instillation model as above, has also been monitored in mice after up to 4 weeks post exposure. In this quantitative study, it was found that about half of the instilled graphene dose has been excreted through the gastrointestinal tract and in spite of the fact that about the same amount was persistently retained in the lungs even after 4 weeks, it caused only a transient dose-dependent pulmonary inflammation and oedema. Interestingly, minor amounts of graphene (up to 1\%) bypassed the alveolo-capillary barrier and were deposited in the liver and spleen [67].

It is important to stress here that in several related studies discussed above the authors used different ways to estimate the exposure dose (e.g. weight by volume and dose per animal), which complicates the direct comparison of the achieved experimental results, clearly strengthening the pressing need on the researchers in the area to reach an agreement on a long-awaited unified approach to dose-exposure calculations in nanotoxicology studies, including those involving graphene-based materials.

\subsection{Systemic parenteral exposure}

The investigations into the possible manifestations of toxicity following this particular exposure route is of a paramount importance for the development of innovative diagnostic imaging probes, therapeutic and multifunctional theranostic nanodrugs alike containing graphene in any form.

An earlier report by Wang et al addressed the issue of acute and chronic dose-dependent toxicity of graphene oxide in mice after systemic intravenous administration. In doses up to $0.25 \mathrm{mg}$ per animal no signs of acute or chronic toxicity were detected. However, the high dose of $0.4 \mathrm{mg}$ per animal $\left(\sim 20 \mathrm{mg} \mathrm{kg}^{-1}\right)$ lead to the accumulation of graphene in the lungs, liver, spleen and kidneys as a result of insufficient clearance, along with the chronic toxicity manifestations, such as lung granuloma formation [68]. In a similar study and equivalent high doses of $20 \mathrm{mg} \mathrm{kg}^{-1}$, pristine few layered graphene and its carboxyl- and PEG-modified forms were predominantly retained in the same organs with no accumulation in the brain, heart or testes after a 3 month monitoring. Irrespective of the surface chemistry, graphene materials accumulation in the organs led to the notable cellular and organ damage manifesting in appearance of necrotic and fibrotic foci as well as glomerular filtration dysfunction [69].

More recently, similar results have been obtained using the intravenously injected graphene oxide, in this case functionalized with poly sodium 4-styrenesulfonate. Graphene material accumulated in the lungs, liver and spleen, was retained in these organ locations 
for over 6 months and induced clear systemic signs of chronic organ-specific inflammation [70]. In contrast, reduced graphene oxide following intravenous injection in rats at a dose of $7 \mathrm{mg} \mathrm{kg}^{-1}$ (comparable to the study above if recalculated to an average rat weight) did not induce any significant changes in haematological, histopathological, liver- and kidney-specific biochemical tests and genotoxicity indicators $7 \mathrm{~d}$ post treatment. Transient fluctuations in the blood leukocyte counts and increased superoxide dismutase activity were the only parameters moderately altered by reduced graphene exposure [71]. A more complex form of graphene such as manganese-intercalated graphene nanoparticles functionalized with dextran intended for the use as magnetic resonance (MRI) contrast imaging agent injected 3 times a week over 3 weeks has been investigated along the lines of dose-dependent subacute toxicity in rats. It has been established that the systemically administered doses not exceeding $50 \mathrm{mg} \mathrm{kg}^{-1}$ of this graphene formulation could be safely used as MRI diagnostic probes [72]. The findings of such extended studies are of prime importance to the development of graphene-enabled nanomedicinal products intended for systemic use, as the results of short-term investigations of graphene oxide biodistribution [73] can possibly lead to overoptimistic interpretations based on the absence of acute toxic effects.

A different, intraperitoneal parenteral administration route has been used by Kurantowicz et al in their comparative investigation of biodistribution of a range of carbonaceous nanomaterials, including graphite and graphene oxide in Wistar rats. The nanomaterials in the form of large aggregates were detected at the injection site as well as in the gastric serous membrane, within the connective tissue of the abdominal skin, muscles and peritoneum. Small aggregates of graphite and graphene oxide nanoparticles were observed in the mesentery and in the connective and lipid tissues near the liver and spleen serosa. None of the tested nanoparticles affected any key blood parameters or growth of rats even at $4 \mathrm{mg} \mathrm{kg}^{-1}$ dose injected 8 times over 4 weeks [74]. In contrast, a comparative study performed in BALB/c mice using intraperitoneally injected pristine graphene, graphene oxide and single wall carbon nanotubes at the equivalent dose of $4 \mathrm{mg} \mathrm{kg}^{-1}$ over $7 \mathrm{~d}$ revealed the increased levels of malondialdehyde and reactive oxygen species and associated morphological damage signs in kidneys and brains of the exposed animals, with the pristine graphene being less toxic than other carbonaceous nanomaterials under investigation [75].

However, despite being important observations from the mechanistic and experimental perspective, this delivery route is one of the least likely to be implemented in humans.

\subsection{Enteric route}

The consequences of oral administration of graphene oxide have been studied in Sprague-Dawley rats at the doses of $10-40 \mathrm{mg} \mathrm{kg}^{-1}$ [76]. The results demonstrated the increased activities of superoxide dismutase, catalase and glutathione peroxidase in a dose-dependent manner in the rat kidneys, along with the significantly elevated serum creatinine, blood urea levels and enhanced accumulation of hydrogen peroxide and lipid hydro peroxide. Altogether, this is a clear indication of the oxidative stress-mediated in vivo nephrotoxicity in experimental animals justifying further in-depth studies in this direction. Ingestion of reduced graphene oxide by mice has also led to transient changes in behavioural activity of the animals, such as general locomotory activity, sense of balance and neuromuscular coordination, which the authors attribute to the possible influence of reduced graphene oxide exposure on the activity of serum superoxide dismutase. In contrast, only little change in exploratory, anxiety type, learning or memory behaviours has been noted [77]. Unfortunately, from these reports it is hard to provide direct extrapolations to the potentially envisaged exposure doses either from the human occupational or consumer products' perspective.

An important study for the first time has been conducted to establish the effects of daily intragastric administration of multi-layered pristine graphene into mice over 4 weeks. In the view of the antibacterial activity of graphene materials discussed earlier in this review, it was very interesting to see that graphene exposure actually increased biodiversity of gut microbiota and changed the microbial community in favour of G-bacteria, possibly due to the selective sensitivity of different microorganisms to oxidative stress and their ability to maintain cell membrane integrity following contact with graphene. As a matter of serious concern, graphene exposure also significantly increased the abundance and types of antibiotic resistance genes in murine gut microbiota [78]. These findings must be undoubtedly taken into account in the design of the new drugs containing graphene intended for oral administration and we can expect more vital discoveries in this field in the future.

\subsection{Transcutaneous (dermal) exposure}

A growing number of recent publications suggests the use of graphene-based materials in innovative dermally applied sensors, diagnostic and transcutaneous drug delivery devices, skin tissue engineering and regenerative systems $[8,79-84]$. Despite the fact that carbonaceous nanomaterials in general, are well known to be associated with the increased incidence of various skin diseases, such as dermatitis, hyperkeratosis, naevi, excessive sensitization and other conditions [85-87], a surprisingly limited number of studies actually addressed the effects of graphene on the dermal toxicity and the functional state of its cellular components in vivo.

Among these, there is a single report that graphene oxide-incorporating antibacterial cotton fabrics, despite being over $90 \%$ lethal to bacteria even 
after a hundred washes, caused no irritation to rabbit skin [88].

The studies on the effects of graphene and its derivatives, such as for example PEGylated graphene oxide/ polypropylene fumarate nanocomposites on one of the key cellular skin components - dermal fibroblasts have been done so far only in in vitro systems [56, 89]. In both reports, graphene was found to be non-toxic to fibroblasts. In contrast to these, Wang et al [68] demonstrated that graphene oxide can actually induce decreased adhesion, cytotoxicity and apoptosis of human fibroblasts at the concentrations over $50 \mu \mathrm{g} \mathrm{ml}^{-1}$.

Altogether, this exposure scenario remains largely underexplored and taking into account controversial available experimental evidence, requires further indepth investigations.

\section{Biological mechanisms affecting grahene toxicity at the cellular and organismal level}

\subsection{Biomolecular corona and protein adsorption}

One of the fundamental factors strongly influencing the nanomaterials' biocompatibility irrespective of their ex-synthesis composition is the formation of the so-called 'biocorona' occurring upon their contact with the biological milieu containing a wide range of biomolecules, including an abundant array of proteins [90-92]. Since the original term 'protein corona' has been coined nearly a decade ago, multiple reports have established its significance for the functional behaviour of nanoparticles and their resulting effects at the cellular and organismal level. Depending on the size, nanoparticles can be either surrounded by the biocorona (in its classical interpretation) or themselves deposit as intercalating 'nano-glitter' [93] on the surface of the interacting biomolecules or within the folded protein structures at the sites where the active physicochemical parameters and factors including size, charge, hydrophobicity/hydrophilicity, available chemical groups and bonds favour such interactions [94].

On the other hand, the dynamic composition of the biocorona can be also actively influenced by the introduction of the surface-modifying agents deposited as additional layers over the nanoparticle core. Notably, an example of surface modification resulting in the improved in vitro and in vivo biocompatibility of graphene oxide was identified using poly(acrylic acid) functionalization. Cytotoxicity, platelet depletion, proinflammatory responses and pathological changes in lungs and liver of mice typically induced by graphene oxides were efficiently alleviated by this surface modification in comparison to the pristine or PEGylated graphene oxide nanomaterials both in in vitro and in vivo tests. Such pronounced effects are explained by the authors as likely to be due to the differential compositions of protein corona, especially immunoglobulin $\mathrm{G}$, forming on the surface of nanoparticles and governing their cell membrane interactions and cellular uptake, the extent of platelet depletion in blood, thrombus formation under short-term exposure and the known pro-inflammatory effects after long-term exposure [95]. These data are in concert with the findings illustrating that BSA protein coating can mitigate the cytotoxicity of graphene oxide by reducing its cell membrane penetration via weakening the interaction between the phospholipids and graphene surface due to a reduction of the available surface area and unfavourable steric effects, thereby significantly reducing the graphene membrane penetration and associated lipid bilayer damaging [96]. Similarly, a strong protein adsorption through $\pi-\pi$ stacking interactions between graphene oxide and aromatic protein residues in addition to hydrophobic interactions was established in a simulation-based molecular dynamics approach. This highlighted a hypothetic potential for reduced cytotoxicity of graphene oxide and reduced graphene oxide nanosheets following their coating with major high abundance blood proteins, which has been subsequently experimentally confirmed [52, 97].

However, the question of the definitive effect of the biocorona as a function of its composition on the graphene toxicity remains not fully understood and a subject of controversy. Thus, the opportunities for the reduction of graphene oxide nano-sheets toxicity have been also addressed in the context of the potential biocorona-contributing secreted compounds found in zebrafish culture water, such as small organic molecules, proteins, nucleotides and mucopolysaccharides. However, in this case the complexes of these biological secretions with graphene displayed a modified nanoplateles topography with thicknesses of about $10 \mathrm{~nm}$ and lateral lengths ranging from 19.5 to $282 \mathrm{~nm}$ exhibiting a more negative surface charge, lower aggregation state and higher toxicity resulting in death, congenital malformations, upregulation of beta-galactosidase and loss in mitochondrial membrane potential of zebrafish embryos [98].

\subsection{Biodegradation, catabolic pathways and clearance}

Since the first encouraging report by Kagan et al in 2010 [99] on the possibility of carbon nanotubes catalytic degradation in vitro and in vivo by neutrophil myeloperoxidase, an intriguing question now is whether the same phenomenon is applicable to a range of other carbonaceous nanomaterial representatives, including graphene in the focus of this review. If yes, it might constitute a valid approach to mitigation of longterm toxicity due to the nanomaterial accumulation, as an efficient elimination mechanism in combination with other biologically important factors, such as 'coronation' with proteins, lipids, and other biomolecules affecting the enzymatic degradation process, as just reviewed in [100].

The uptake and potential signs of degradation of carbonaceous nanomaterials, including graphene 


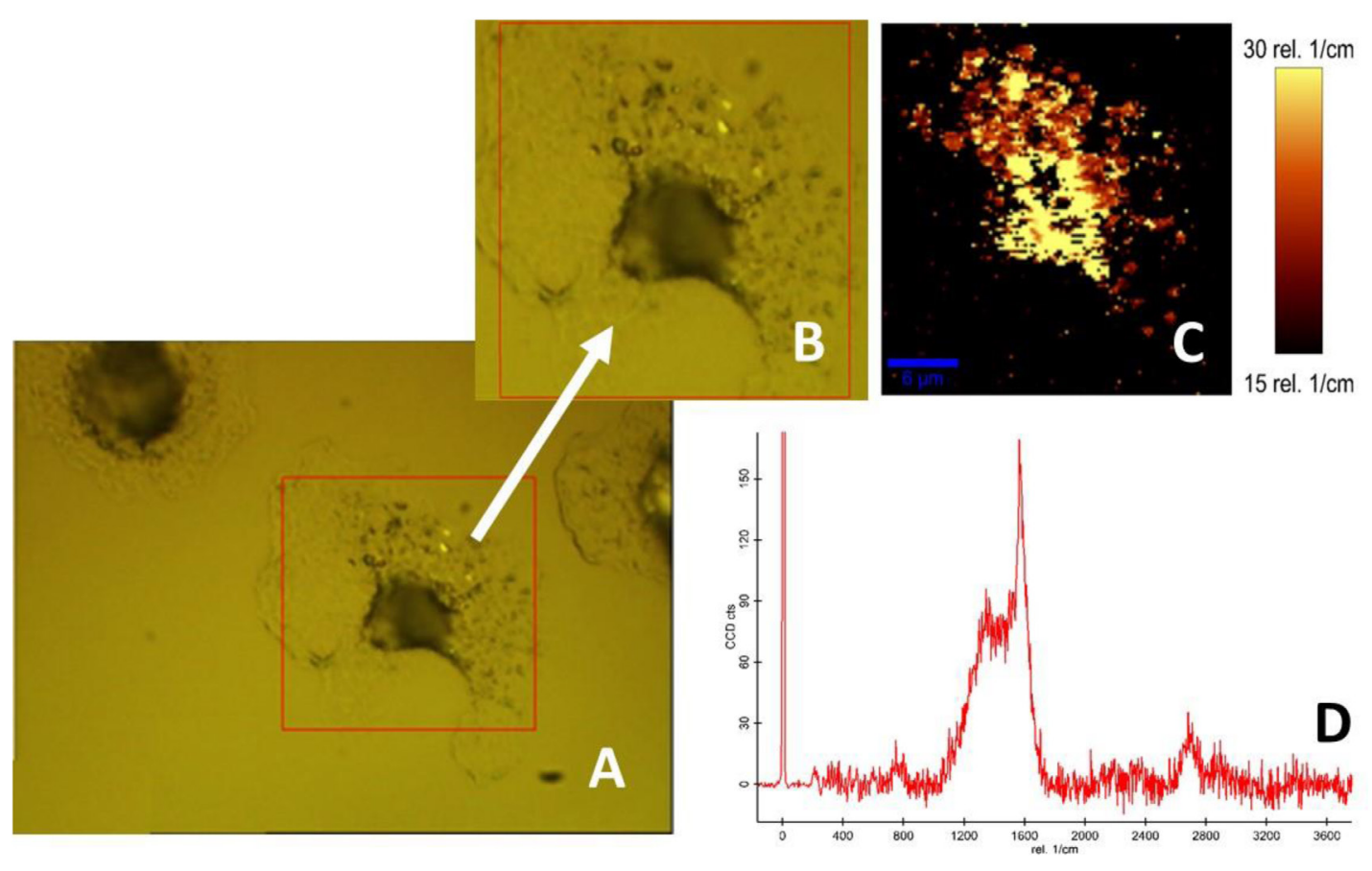

Figure 3. Pristine graphene uptake by phagocytes analysed by Raman spectroscopy. (A) Transmitted light image of primary human macrophages exposed to pristine graphene flakes. (B) The microscopic field selected for Raman mapping. (C) G-band $\left(\sim 1580 \mathrm{~cm}^{-1}\right)$ Raman mapping over the selected cell area, full width at half maximum (FWHM) measurement. (D) A representative Raman spectrum of graphene within the macrophage. The presence of D-band $\left(\sim 1350 \mathrm{~cm}^{-1}\right)$ in graphene can serve as an indicator of the appearance of disordered structure and hence possible biodegradation. The details of the experimental procedure are fully explained in [54] published by the authors of this review. The illustrations correspond to the figure 10 of the publication, but were not selected for the original manuscript from the repeated consistent sets of experimental data and hence were not previously subject to copyright.

within the cells can be can be analysed in a very specific quantitative manner by Raman spectroscopy (figure 3), as the appearance of a D-band $\left(\sim 1350^{-1}\right)$ can serve as an indicator of the material structural disintegration and biodegradation potential $[69,101]$.

Catabolic degradation routes triggered in phagocytes after carbonaceous nanomaterials exposure have been very recently addressed by McIntyre et al [54]. The authors compared the catabolic processes induced in primary human macrophages by pristine graphene and pristine single walled carbon nanotubes, both with similar surface chemistries but different geometries. It was shown that the PG did not behave like any of its derivatives, it was phagocytosed by the primary macrophages in abundance, did not induce autophagy and was not degraded following $24 \mathrm{~h}$ accumulation within these cells. Of an important note, graphene oxide in contrast to the above observation with pristine graphene, has been shown to be more prone to induction of the autophagic type of protective anti-toxic response in human-hamster hybrid mammalian cells against polychlorinated biphenyls, acting as their potential adsorbent due to its large surface area and high abundance of oxygen-containing functional groups [102].

This makes us put a strong note that despite the fact that biodegradation of graphene and graphene-based products is an important and potentially exploitable pathway of alleviating the toxicity of these nanomaterials, it must be kept in mind that the biodegradation process per se can result on the formation of more chemically aggressive derivatives possessing strong adverse effects on the normal body cells and tissues. The recent overview by Vlasova et al [103] witnesses that a plethora of diverse oxidative enzymes present in inflammatory cells, such as myeloperoxidase, peroxidase of eosinophils, lactoperoxidase, xanthine oxidase and haemoglobin, all of which can contribute to the biodegradation of nanomaterials. Such widespread oxidative machinery applied to graphene can in principle cause the formation of the oxidized species with even more pronounced toxic characteristics than those of the original nanomaterial [104].

The issue of a paramount importance related to the nanomaterials with limited or not fully identified biodegradation pathways is the degree of biopersistence and clearance of these materials from the organism following the exposure resulting in systemic distribution. On one hand, it is dictated by the size of the nanoparticle in question which must not exceed the filtration threshold of the kidney barrier of 10-12 nm $[105,106]$ and should be not subject to increase due to the aggregation prior to reaching the filtration barrier. Two recent studies carried out by Jasim et al addressing the biodistribution and urinary clearance of graphene oxide sheets of different thickness following systemic intravenous injection $[73,107]$, demonstrated that even with the lateral dimensions falling in the micrometre range, graphene oxide materials with an average thickness of 2-10 nm can still successfully pass though the murine kidney filtration barrier, therefore apparently 
lending themselves to convoluted geometrical shape adaptations under the physical shear forces involved in the process. Nonetheless, the report by Sasidharan et al [69] denies any appreciable renal clearance of few layer pristine graphene or its carboxylated and PEGylated derivatives even of the much smaller average thickness of $0.8-1.3 \mathrm{~nm}$ and lateral dimensions of $115-160 \mathrm{~nm}$, leaving the issue of clearance of graphene materials via renal filtration a subject of controversy. On the other hand, the efficiency of the clearance is also dramatically affected by the thickness- and surface functionalisation-dependent graphene nanoparticles retention in the cells of the reticuloendothelial system of the parenchymatous organs such as spleen and liver [107] and/ or associated secondary inflammatory effects in other organs, e.g. lungs [66]. As a result, the success on the front of construction of clearable carbonaceous nanomaterials in general and graphene-enabled medicinal products in particular, is so far very limited, with just a few publications reporting such possibility [73, 107, 108-110], therefore justifying further expanded investigations into this field.

\section{Approaches to toxicity mitigation and safe-by-design development of graphene-enabled products}

\subsection{Risk assessment strategies}

The introduction of graphene-enabled products (GEP) into the market has to be appropriately assessed in terms of the associated risks and uncertainties at macro-, micro- and nano-scale level. In particular, before novel materials and products will be placed on the market it is crucial to minimise potential risks regarding development costs as well as regulatory needs and environmental concerns (i.e. health and safety aspects).

Since graphene as advanced material lends itself to a large variety of different applications, it provides a challenge for assessing their risks and associated safety for consumer, occupational workers and the environment. Furthermore, as presented above, depending on the end-use application GEP can have many physicochemical property differences. Therefore GEP risk assessment can be extremely demanding if for each product resources and time have to be committed in a case-by-case basis. Therefore, most of the scientific, industrial and regulatory drive in the past 10 years has been focused on developing suitable characterisation cascade, quality assurance tools and frameworks for risk assessments, management and monitoring of emerging products containing advanced materials. In most of the cases the enhancement is associated with a nanoscale property of the materials in use [111].

The introduction of a tiered safe-by-design approach [112] can provide a de-risking strategy towards the identification of uncertainties and risks from the early developmental stages of GEP. Standardisation of graphene characterisation is also an important aspect towards the increased consumer confidence, reproducibility, and quality assurance of various types and formulations of carbon products. Furthermore, the introduction of safety thresholds of each components of the GEP will allow for a prioritisation in the risk assessment process. There highest health risks are the first to be screened and this is where the most important information is acquired and assessed. Such approach therefore allows for the creation of nano-specific risk assessment strategy, as proposed recently by Dekkers et al [113] which can be applied across the different industries for the benefit of the consumer, and in the case of medical products, for the patients.

\subsection{Selecting the optimal physicochemical characteristics}

Several important considerations must be taken into account when attempting to reduce the toxicity associated with exposure to GEP and to implement appropriate risk mitigation measures. Such fundamental physical characteristics as size [56, 114], shape and geometry $[18,20,27]$, charge $[26,27,56]$ and dispersion/aggregation state $[37,66,115]$, can be effectively used to identify and exploit the optimally performing graphene-based systems and devices for a particular intended application with minimal healthdeteriorating side effects. A significant impact can be also imposed by the difference in the toxicity potential of pristine, oxidised and reduced forms of graphene, with less oxidized graphene reported to produce higher levels of reactive oxygen species, cytotoxicity and apoptosis [104]. Among the most recent and appealing findings, we must also mention the opportunity to exploit chiral properties of graphene quantum dots. The exposure of liver HepG2 cells to the L/D-cysteine moieties attached to the edges of graphene quantum dots revealed their general biocompatibility and a noticeable difference in the toxicity of such stereoisomers [116].

\subsection{Surface modification by chemical functionalisation}

Among the earlier reported simple approaches to reduce damaging effect of graphene oxide on red blood cells, the coating with chitosan has been demonstrated as a nearly 100 percent efficient way for elimination of this type of toxicity [56]. A pluronic block copolymer in complex with graphene oxide has been shown to significantly reduce the toxicity of graphene oxide in human fibroblasts and demonstrated the potential of this approach for everyday life bacterial disinfection applications, as hypotonic pluronic and graphene oxide mixture proved to be both safe and effective [52, 117].

In one of the most recent studies a new unique hydroxylated graphene derivative has been shown to provide a beneficial environment for cell adhesion and growth of rat adipose tissue-derived stromal cells due to its hydrophilicity and weak inductive nature. Of note, the overall simplicity of this approach enables to produce such graphene derivative in industrial 
kilogram-scale amounts [84]. Graphene nanoplatelets surface-modified with polyvinyl acetate rendered them non-toxic at the concentrations of up to $50 \mathrm{mg} \mathrm{ml}^{-1}$ compared to pristine nanoplatelets in HFF-1 cells model [118]. Amine-modified graphene oxide has been proven to be a much safer functionalised material in terms of the potential thrombogenic and haemolytic effects in mice in vivo in comparison to its unmodified graphene oxide or reduced graphene oxide counterparts [119].

\subsection{Other exploitable environmental and biological factors}

Humic acid as a natural organic matter has been found to be an effective graphene oxide toxicity alleviation agent in E. coli aqueous bacterial model. This natural agent exhibited an antioxidant action role, maintaining the activity of the antioxidant enzymes and decreasing the reactive oxygen species generation, as witnessed by the results of oxidative stress experiments, thereby having important ecotoxicology implications [120].

A very appealing observation from the microbiological point of view has been made by Zhao et al who have found that the pre-treatment with the established famous probiotic Lactobacillus bulgaricus prevented graphene oxide toxic effects on the functions of both primary and secondary targeted organs in wild-type nematodes Caenorhabditis elegans and reduced graphene oxide damage in nematodes with mutations of susceptible genes (sod-2, sod-3, gas-1, and aak-2) by sustaining normal intestinal permeability. L. bulgaricus also sustained the normal defecation patterns in both wild-type nematodes and those with mutations of susceptible genes. The beneficial role of L. bulgaricus against graphene toxicity is explained by the authors as due to the combinational effects on intestinal permeability and defecation behaviour. Interestingly, the beneficial effect of L. bulgaricus on graphene toxicity was dependent on the function of acs-22 gene homologous to mammalian fatp 4 encoding the fatty acid transport protein 4 . This study, despite been carried out in worms, might pave the way towards innovative pharmacological strategies to protect a natural intestinal barrier from the graphene oxide toxicity in higher species [121].

Another novel and attractive 'all-biological' approach for assembling water-soluble and cellcompatible graphene oxide has been offered using Ginkgo biloba extract as a reducing and stabilizing agent, resulting in the overall lower toxicity and increased biocompatibility of the complex [122].

\subsection{Facilitated degradation}

The rationally designed graphene oxide nanocarriers modified with polyethylene glycol and branched polyethylenimine have been introduced to control the biological activity of oxidised graphene as a nanodrug carrier and its degradation in biological systems. Such nanostructures efficiently interact with plasmid DNA forming a stable nanocomplex via electrostatic interactions. Following the uptake by the cells, the complex can readily escape from endosomes by photothermal conversion of graphene oxide upon near-infrared irradiation and photothermally induced endosome disruption. As a result, reducing intracellular environment enables polymer dissociation and rapid gene release with enhanced transfection efficiency and decreased toxicity in comparison to non-reducible amide-functionalized graphene nanocarriers. In addition, the de-PEGylated graphene oxide nanocarriers exhibit higher engulfment by phagocytes due to their exposed disulfide bonds, and are subject to subsequent facilitated degradation in macrophages [123]. An innovative approach was developed by Zan et al to fabrication of water-dispersible nanocomposites with iron oxide nanoparticles attached to graphene, enabling to produce biocompatible and apparently biodegradable structures intended as potential sensitive T2 contrast agents. The authors claim that these composites can be cleared from the body through the metabolic processes and therefore are harmless to the living organism [124]. However, as the biodegradation process in this case was related only to the iron oxide component of the complex, this study reinforces the importance of implementation of the above mentioned safe-by-design approach [113], which must be strictly adhered to from the very early stages of the new nanomedicines development in relation to all the constituent components. It ensures that the new diagnostic and therapeutic preparations are safe, have maximal possible specific efficiency with minimal impact on the normal cells and therefore can eventually find their approved clinical applications and earn the deserved space on the pharmacy shelves.

\section{Emerging opportunities for biomedical applications}

\subsection{Graphene-enabled products as bactericidal agents}

In the general context of potential graphene toxicity to living biological systems, the established toxic effects displayed against pathogenic bacteria discussed above could constitute a desirable and exploitable property. However, overall the bactericidal activity of pristine graphene and graphene oxide materials is known to be relatively low, which in the view of potential human exposure significantly reduces benefit-to-risk ratio. Hence, most recent reports have been largely focussed on investigation of graphene-based nanomaterials with various surface functionalities or in the form of composites enabling to boost the antimicrobial efficacy.

Graphene oxide in a nanocomposite complex with silver has demonstrated a remarkable bactericidal activity to some of the most common hospital contaminants including Acinetobacter baumannii, Enterococcus faecalis, and E. coli and even to such a notorious strain as methicillin-resistant S. aureus (MRSA) [125]. A relatively short $4 \mathrm{~h}$ exposure to graphene-Ag nanoparticles 
lead to a complete growth suppression of pathogenic bacteria via a direct contact mechanism, compared to the graphene oxide or silver nanoparticles alone. In a related study, a systematic analysis of antimicrobial activity of several graphene and heavy metal nanoderivatives such as graphene oxide nanosheets, Ag and $\mathrm{Cu}$ nanoparticles and their nanocomposite combinations against E. coli, Pseudomonas aeruginosa, S. aureus, Klebsiella pneumoniae and methicillin-resistant $S$. aureus has been carried out. Graphene oxide in a composite with $\mathrm{Cu}$ and $\mathrm{Ag}$ displayed a clearly enhanced wide spectrum bactericidal effect, however to a lower extent detectable in MRSA [126].

An innovative route to increase the antibacterial effects of graphene oxide has been demonstrated by Buccheri et al [127]. This group used laser irradiation to attenuate the targeted properties of this nanomaterial. Antibacterial properties of laser radiation-modified graphene oxide investigated in E. coli have been found to be higher for graphene oxide irradiated for over $3 \mathrm{~h}$, which led to the reduction of the nanographene flakes size whilst retaining a significant oxygen content and hydrophilic properties of the material. Bacterial cell walls shown a marked degree of damage following the exposure to the irradiation-modified graphene oxide. In the same study, the authors carried out tests in zebrafish embryos and came to the conclusion that that neither mortality nor sublethal effects were induced by laser-irradiated graphene oxide flakes in the concentrations of up to four times exceeding those which were found effective in lowering the bacterial growth.

A more generalised approach to increase the above mentioned benefit-to-risk value of graphene oxide has been offered by Karahan et al [117]. They used an abrupt change in the environmental salinity or water shock treatment of bacteria with a subsequent treatment with the complexes of graphene oxide and pluronic F-127 block copolymer known for its good biocompatibility characteristics. As a result, in under $3 \mathrm{~h}$ nearly $100 \%$ of the bacteria with compromised cell walls exposed to such nanocomplexes were killed, compared to under 30\% bactericidal efficiency registered without such treatment. Pluronic-graphene complexes at the same time displayed a reduced toxic effect on normal human fibroblasts.

An example of a thorough systematic approach to investigation of biological effects of more complex graphene derivatives on microorganisms as a function of their structural and functional properties has been shown by Diez-Pascual and Diez-Vicente [89]. In this report incorporating an extensive physicochemical characterisation of a nanocomposite material based on the PEGylated graphene oxide and polypropylene fumarate, the authors evaluated the microbicide efficiency of nanocomposites against several types of G-positive and G-negative bacteria, such as S. aureus, Staphylococcus epidermidis, $P$. aeruginosa and E. coli. They have found a remarkable degree of antimicrobial activity of graphene oxide-based composites which was developing in a dose-dependent manner with increasing concentrations of graphene. Of note, the authors found that G-positive bacteria responded in a more pronounced manner to such treatment. Despite being important phenomenological observations, the specific mechanisms affecting the sensitivity of diverse bacterial types to graphene exposure apart from the presence or absence of outer membrane are yet to be disclosed.

\subsection{Graphene-enabled products for cancer treatment}

Cancer, as a disease of a prime socioeconomic importance, has been attracting the efforts of researchers working with advanced nanomaterials for a number of years. This is not surprising firstly because the arsenal of available treatment strategies is still very limited and none of the currently available medications can guarantee a complete cure without the serious side effects. Secondly and partially due to the above, the pathway for the regulatory approval of new anti-cancer drugs could be faster and more straightforward than in application to other diseases. Thirdly, nanomaterials possess a number of unique properties bringing them to the forefront of potential cancer diagnostics and drug delivery systems, such as the opportunity to exploit the EPR (enhanced permeability and retention) effect $[128,129]$ attributed to tumours, capability to deliver a high localised drug load and theranostic multifunctionality. Applied to the malignant cells, the term 'cytotoxicity' largely acquires the meaning of 'anticancer activity' and constitutes a desired effect. In an ideal case scenario, the new nanomaterial intended for cancer treatment should be cytotoxic to malignant cells and exert minimal or no effect on the surrounding normal cells and tissues. Unfortunately, such material ex synthesis does not yet exist and therefore the search for the appropriate surface functionalisation of nanomaterials enabling to ensure such selectivity continues worldwide. Over the last few years, several promising graphenebased nanotools have been developed and demonstrated a strong potential for cancer therapy applications.

Thus, the exposure to graphene oxide and reduced graphene oxide produced using uric acid (UA-rGO) resulted in a dose-dependent decrease in cell viability and induced cytotoxicity in human ovarian cancer cells. The results of this study indicate that UA-rGO could trigger apoptotic mechanisms in malignant cells. The anti-cancer cytotoxic effects of UA-rGO were significantly higher than those of the graphene oxide and involved increasing lactate dehydrogenase (LDH) release, reactive oxygen species generation, activation of caspase-3, and DNA fragmentation [130].

Graphene oxide and its derivatives dodecylamine graphene oxide, reduced graphene oxide and sodium dodecyl sulfate graphene oxide displayed a strong cytotoxic activity against the cells of a human lung 
carcinoma line A549 in a concentration-dependent manner starting with moderate effect at 3-25 $\mu \mathrm{g} \mathrm{ml}^{-1}$ and reaching a dramatic cell viability reduction at 50-400 $\mu \mathrm{g} \mathrm{ml}^{-1}$ [131]. In another approach, a complex original hybrid nanostructure comprising graphene oxide and $\mathrm{Zn}$-clinoptilolite as a potential drug carrier has been explored in the same cancer cell line A549 for its biocompatibility and it has been shown that this construct possessed low baseline toxicity and high anticancer drug doxorubicin loading capacity, making it a potentially good drug nanocarrier candidate [132].

An innovative system comprising hollow magnetic nanospheres coated with the silica shells and conjugated with carboxylated graphene quantum dots as a core-shell composite has been offered for multimodal cancer treatment. The composite was further loaded with doxorubicin and stabilized with liposomes. This multimodal system was able to destroy cancer cells by four different therapeutic mechanisms in a synergetic and multilateral fashion, namely, the magnetic field-mediated mechanical stimulation, photothermal damage, photodynamic toxicity and chemotherapy. The registered combined effect was dramatically higher than any of those attributed to the individual component therapeutic modalities [133].

Graphene oxide functionalized with urease B as the model antigen has been recently implemented as a vaccine adjuvant for immunotherapy acting as a positive modulator to promote maturation of dendritic cells and to enhance their cytokine secretion through the activation of multiple toll-like receptor pathways while showing low toxicity. Further in vivo studies confirmed that this engineered graphene oxide derivative was more efficient than free urease B or clinically used aluminum-adjuvant-based vaccine in induction of cellular immunity, suggesting its promising potential for cancer immunotherapy [134].

In a comparative investigation by Piper et al of graphene oxide containing variable redox-active groups on the surface, including manganese ions, C-centered radicals and endoperoxides, the latter were found to be the most potent in generating reactive oxygen species and associated toxicity in cultured human cervical cancer cells [135].

A new range of exciting opportunities in the area of cancer treatment has been offered by the report by Fiorillo et al [136] who convincingly demonstrated in the advanced tumour spheroid models that graphene oxide could be utilised for cytotoxic targeting of cancer stem cells of different types, including some of the most notorious malignant tumours such as pancreatic cancer and glioblastoma. Remarkably, the authors made a significant departure from the phenomenological observations, providing an insight into the mechanistic elements of the findings and identifying Wnt, Notch and STAT-mediated signalling pathways as key contributors into the induction of cancer stem cells differentiation, paving the way to the development of new selective anti-cancer nanomedicines.

\subsection{Advanced multimodal medical applications of polyfunctional graphene derivatives}

A series of recent publications addresses the measures for improved biocompatibility and lowered toxicity using more complex functionalisations of graphene, which is of a particular importance for the prospective diagnostic imaging, therapeutic and multimodal theranostic tools. For example, indocyanine greenloaded polydopamine-reduced graphene oxide nanocomposites with amplifying photoacoustic and photothermal effects enabled to achieve a complete suppression of tumors in 4T1 breast subcutaneous and orthotopic mice models after photoacoustic imaging-guided photothermal treatment, with no signs of accompanying systemic toxicity [137]. A similar approach using indocyanine green conjugated with PEGylated reduced graphene oxide has been implemented for photoacoustic and fluorescence dualmodality tumour imaging in vivo in mice. The resulting nanocomposites had minimal toxicity and superior passive tumour targeting with steady photoacoustic and fluorescence signals sustained over $6 \mathrm{~h}$ post systemic intravenous injection [138].

Luo et al have constructed a related polyfunctional nanosystem integrating the photodynamic and photothermal therapy for cancer treatment. In their approach, a photosensitizer IR-808) with cancer-targeting ability and near-infrared sensitivity was chemically conjugated to both polyethylene glycoland branched polyethylenimine-functionalized graphene oxide nanoparticles. A significantly enhanced photodynamic and photothermal therapy effects were achieved both in murine and human cancer cell models apparently facilitated by the preferential accumulation of the nanocomplexes by cancer cells mediated by the organic-anion transporting polypeptides commonly overexpressed on malignant cells surface. In this case, it was possible to achieve a complete tumour ablation in two xenograft models with no detectable toxicity in comparison to controls [139].

Additional opportunities in this direction are offered by introduction of specific targeting moieties, which allow for the localised accumulation of the graphene-incorporating theranostic nanotools, thereby dramatically reducing the side effects of the loaded chemotherapeutic agents. Thus, the efficient use of graphene oxide which was conjugated to a targeting monoclonal antibody against follicle-stimulating hormone as a highly selective tumour vasculature marker, has been shown in a murine model of breast cancer metastasis assisted by bioluminescence imaging. Histological analysis confirmed the active vascular accumulation of these conjugates in lung metastatic nodules and tumours at early time points. On top of this, such targeted graphene oxide conjugates are promising for the use as theranostic tools due to a good anti-cancer drug loading capacity [140]. Another group of authors has successfully utilised new somatostatin receptor-mediated tumour targeting nanotools based on octreotide-PEG conjugated 


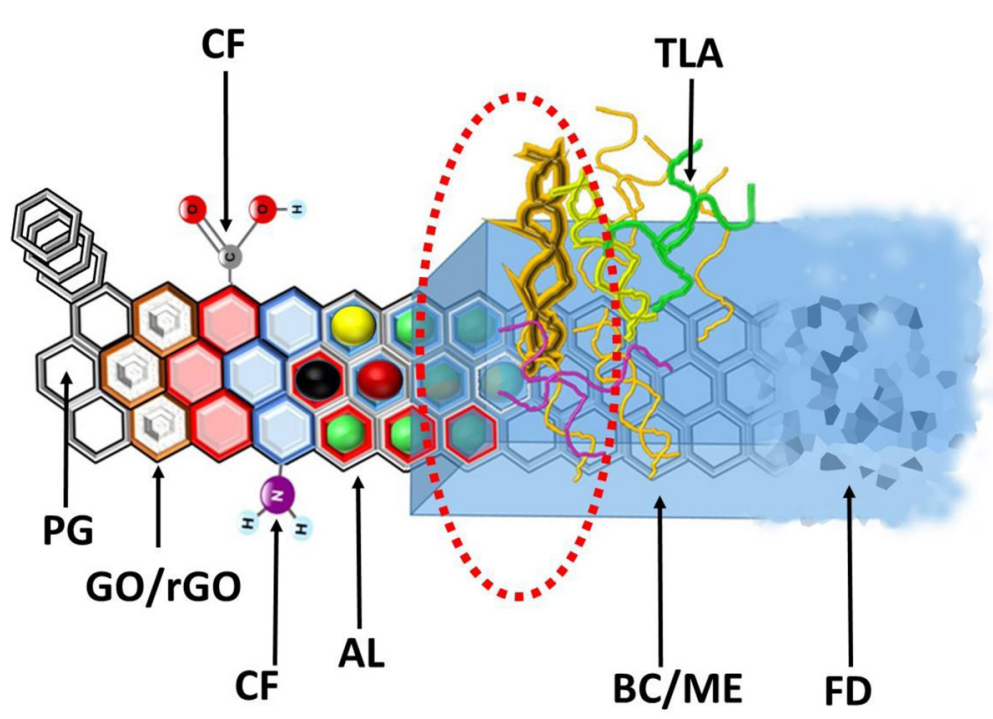

Figure 4. The key factors affecting the toxicity of graphene, graphene-enabled products and opportunities for their targeted functionalisation towards biomedical applications. PG_-pristine graphene with varying number of layers (thickness), lateral dimensions and sharpness. GO/rGO_graphene oxide/reduced graphene oxide. $\mathrm{CF}$ —chemical functionalisation (e.g. amine and carboxyl groups). AL_accessory loading with magnetic nanomaterials (black spheres), photosensitizers (red spheres), lightemitting probes (yellow spheres) or biologically active compounds, e.g. anti-cancer drugs (green spheres). BC/ME-biocompatible coating and/or matrix embedding (e.g. polymers). TLA — targeting ligands addition (proteins, antibodies, peptides etc). FG-facilitated degradation (artificially induced and/or bio-degradation). The area marked by red dotted line denotes the structure with several functionalities present at a time, which could be exploited in theranostic multimodal applications. Source: an original illustration by the authors.

graphene oxide for combined chemo- and photothermal therapy. These nanotools showed low systemic toxicity, high efficiency of photothermal tumour ablation and improved localised precision delivery of anti-cancer drug doxorubicine [141].

The work of Zhou et al demonstrated that through the implementation of bio-responsive nanosystems it is possible not only to increase the efficacy of anti-cancer nanodrugs, but also to significantly suppress the undesirable toxic side effects. They have constructed a multifunctional construct incorporating graphene oxide with transferrin enabling receptor-mediated endocytosis and chemotherapeutic drugs load as therapeutic modalities. This approach enabled to facilitate the cell internalisation, accumulation and cytotoxic activity of the construct against cancer cells, at the same time showing less damaging impact on the normal cells. This multifunctional nanodrug system triggered apoptosis in malignant cells through activation of p53 and MAPK-dependent pathways and was simultaneously leading to suppression of ERK and AKT-mediated signalling [142].

An example of a safe and efficient nanocomplex for colon cancer drug delivery has been presented in the form of a hydrogel designed using $\mathrm{pH}$-sensitive and biocompatible graphene oxide containing azoaromatic crosslinks, polyvinyl alcohol and an encapsulated anti-cancer drug curcumin. The results of the studies in colon-specific drug delivery systems demonstrated that such composite hydrogels can protect the active drug during passing through the aggressive environment of the stomach and small intestine to the proximal colon and to enhance the colon-targeting ability and residence time in the colon, thereby preserving high drug efficiency and maintaining low toxicity [143].

A remarkable stimulating effect on the biocompatibility and metabolic activity has been lately shown in osteoblastic cell line MC3T3 and human mesenchymal stem cells using polyethylene-immobilized, graphene oxide reinforced high-density polyethylene nanocomposites [144]. In concert with these results, it has been shown that graphene oxide-coated substrates stimulated the differentiation of mouse embryonic stem cells to both primitive and definitive haematopoietic cells. Notably, graphene oxide also facilitated human embryonic stem cells differentiation to blood cells thereby offering an intriguing opportunity for developing new strategies towards the generation of large numbers of functional blood cells potentially exploitable in patients with blood disorders or hematologic malignancies [145].

From the critical perspective, however, most of the studies on the advanced multifunctional graphenebased nanosystems suffer a serious drawback as they do not provide an insight into the long-term consequences of these applications intended for systemic administration either due to the discontinuation of the experiments upon reaching the desired registerable effects (e.g. statistically significant tumour volume reduction) or are limited by a natural lifespan of small laboratory animals. This pressing issue undoubtedly must be addressed by the researchers investing their efforts into the development of new generation nanomedicines. 
7.4. Implantable devices and regenerative medicine

This promising area of biomedical applications of GEP has deservedly received a steadily rising attention over the last few years and has been lately extensively covered in specialised topical overviews elsewhere $[146,147]$. However, we can't omit addressing here some of the elegant reports on graphene-enabled products intended to be used as implantable devices and scaffolds in human tissues in vivo. The study which deserves a special attention in this regard implemented electrospun microfibre scaffolds coated with selfassembled colloidal graphene which were implanted into the striatum or into the subventricular zone of rat's brain. Amazingly, graphene coating was associated with anti-inflammatory effects which manifested in reduced microglia and astrocyte activation levels and prevented glial scarring after 7 weeks post implantation. Among the other registered effects were astrocyte guidance within the scaffold and redirection of neuroblasts from the subventricular zone along the implants [148]. This observation has potentially far reaching repercussions in the area of biocompatible sensors development and neural tissue remodelling and regeneration.

Graphene has been also reported to exert beneficial effects in dental and orthopaedic surgery by protecting dental implant surfaces against cariogenic bacteria [149] and stimulating the morphogenetic processes via facilitated osteogenic differentiation of mesenchymal stem cells and surface bioactivity [150, 151], whilst polypropylene fumarate nanocomposites in complex with PEG-functionalized graphene oxide display no cytotoxic effects in human dermal fibroblasts, making them also remarkable candidates for the intended use in orthodontic and general bone tissue engineering [89].

Altogether, the key factors affecting the toxicity of graphene and its derivatives along with the exploitable opportunities arising from selective GEP functionalisation for biomedical uses are summarised and schematically illustrated in figure 4 .

\section{Conclusions and future perspectives}

A detailed analysis of the most recent original research reports along with the earlier review publications unequivocally confirms that graphene in any of its numerous forms and derivatives must be approached as a potentially hazardous material. It exerts its effects on a widest range of living organisms, including prokaryotic bacteria and viruses, plants, micro- and macroinvertebrates, eukaryotic mammalian and human cells and whole animals in vivo. However, a significant discrepancy and frequently even controversy existing between different experimental findings conducted even in closely related models dictates the demand for further more systematic and coordinated multicentre research investigations including a detailed physicochemical characterisation of the specific graphene materials utilised in each study.
Irrespective of the particular type of graphene used, it is largely accepted that the generation of reactive oxygen species lies at the basis of its toxicity, further attenuated by structural and chemical properties of the nanomaterial. On this note, there is a clear difference between the oxidised graphene forms most frequently referred to and the pristine graphene which deserved a much more modest attention, though even from the limited number of comparative observations, it appears to possess far less toxic potential. More extensive studies in this area are therefore necessitated.

Graphene toxicity has been investigated in a variety of systems providing, to a certain extent, the imitation of physiologically relevant exposure scenarios in humans. However, some of the most likely exposure routes remain underexplored, such as for example, the transcutaneous pathway, despite the fact that it could be involved both in everyday life originating from the graphene-containing consumer products and in the future as smart diagnostic devices and sensors developed for dermal application. Therefore, we expect to see further in-depth efforts of scientists in this direction.

With the emergence of new synthetic methods and scale-up manufacturing processes we will undoubtedly witness a steady increase in global graphene production volumes. It is predicted that the graphene market will grow to a total volume sales of 200 million \$ (nearly $3.8 \mathrm{k}$ tonnes per annum) by 2020, according to IDTechEx latest report. On this front, the implementation of effective risk management strategies at the manufacturing, laboratory and clinical settings can minimise the exposure to graphene and its environmental discharge.

The increasing presence of graphene-enabled products on the market also dictates a demand for nanospecific scientific knowledge which has to provide the basis for benchmarking, validation and trusted data for the regulatory acceptance of GEP. However, despite frameworks for acceptance have been developed by now, there is the need to provide further insight into the specific properties that are critical in determining the transformation and behaviour of GEP. These will allow for more efficient risk assessment and management strategies. The introduction of safe-by-design and high benefit-to-risk ratio practices will also accelerate the market approval and applications development for the ultimate benefit of the patients and society.

Furthermore, numerous promising post-synthesis approaches have already been offered targeting the opportunities of efficient reduction of graphene hazardous features. Surface passivation, charge manipulation, and introduction of biocompatible coatings are just a few to list. Exploiting artificially induced degradation and/ or naturally operating catabolic pathways of graphene represents another promising and intriguing window of opportunity and it is of a particular importance in relation to bio-persistence and long-term toxicity of this nanomaterial, since the extended (over several months) in vivo monitoring studies in the area are extremely scarce. 
On another positive note, the double-edged sword of graphene toxicity could be efficiently used for exploitable beneficial opportunities. The established antimicrobial activity of graphene can be utilised for generation of new nano-enabled graphene-based disinfectants and bactericidal coatings with maximal efficacy to exposure ratio and it is likely to be offered not in a very distant future. Anti-cancer properties of graphene nanomaterials, including selective cancer stem cell targeting and a stream of very exciting studies demonstrating the possibilities of generating polyfunctional graphene-based complexes for multimodal theranostic applications lets us have an optimistic outlook on the development of highly innovative nanotools for cancer diagnostics, treatment and disease progression monitoring in the future.

We strongly believe that further consolidation of the results of the new imminent in-depth studies in the unified graphene materials toxicity database reinforced by the implementation of responsible manufacturing and laboratory practices can contribute to the significant reduction of risks inflicted by the potential graphene exposure and help in establishing an appropriate educated awareness level in this field for the overall benefit to the society.

\section{Acknowlegments}

Supported by the Higher Education Authority of Ireland, Science Foundation of Ireland through the Advanced Materials and BioEngineering Research (AMBER) project (Grant \#SFI/12/RC/2278) and partially by NANoREG project (FP7-NMP2012 Grant \#310584). We thank Tiina O'Neill (Electron Microscopy Core Facility, UCD Conway Institute, UCD, Belfield, Dublin 4) for the TEM samples preparation and imaging and Niall McEvoy (CRANN, Trinity College Dublin) for help with Raman mapping studies.

\section{References}

[1] Das A and Snee P T 2016 Synthetic developments of nontoxic quantum dots ChemPhysChem 17 598-617

[2] Lalwani G, D'Agati M, Khan A M and Sitharaman B 2016 Toxicology of graphene-based nanomaterials Adv. Drug Deliv. Rev. 105 109-144

[3] Syama S and Mohanan P V 2016 Safety and biocompatibility of graphene: a new generation nanomaterial for biomedical application Int. J. Biol. Macromol. 86 546-55

[4] Goenka S, Sant V and Sant S 2014 Graphene-based nanomaterials for drug delivery and tissue engineering J. Control. Release 173 75-88

[5] Ema M, Hougaard K S, Kishimoto A and Honda K 2016 Reproductive and developmental toxicity of carbon-based nanomaterials: a literature review Nanotoxicology 10 391-412

[6] Yang G, Zhu C, Du D, Zhu J and Lin Y 2015 Graphene-like two-dimensional layered nanomaterials: applications in biosensors and nanomedicine Nanoscale 7 14217-31

[7] Gurunathan S and Kim J H 2016 Synthesis, toxicity, biocompatibility, and biomedical applications of graphene and graphene-related materials Int. J. Nanomed. 11 1927-45

[8] Ding X, Liu H and Fan Y 2015 Graphene-based materials in regenerative medicine Adv. Healthcare Mater. 4 1451-68
[9] Orecchioni M, Menard-Moyon C, Delogu L G and Bianco A 2016 Graphene and the immune system: challenges and potentiality Adv. Drug Deliv. Rev. 105 163-75

[10] McCallion C, Burthem J, Rees-Unwin K, Golovanov A and Pluen A 2016 Graphene in therapeutics delivery: problems, solutions and future opportunities Eur. J. Pharm. Biopharm. 104235-50

[11] Jarosz A, Skoda M, Dudek I and Szukiewicz D 2016 Oxidative stress and mitochondrial activation as the main mechanisms underlying graphene toxicity against human cancer cells Oxid. Med. Cell. Longev. 20165851035

[12] Zhang B, Wang Y and Zhai G 2016 Biomedical applications of the graphene-based materials Mater. Sci. Eng. C 61 953-64

[13] Chakrabarti M, Kiseleva R, Vertegel A and Ray S K 2015 Carbon nanomaterials for drug delivery and cancer therapy J. Nanosci. Nanotechnol. 15 5501-11

[14] Schroeder K L, Goreham R V and Nann T 2016 Graphene quantum dots for theranostics and bioimaging Pharm. Res. (doi: 10.1007/s11095-016-1937-x)

[15] Malard L M, Pimenta M A, Dresselhaus G and Dresselhaus M S 2009 Raman spectroscopy in graphene Phys. Rep. 473 51-87

[16] Ferrari A C and Basko D M 2013 Raman spectroscopy as a versatile tool for studying the properties of graphene Nat. Nanotechnol. 8 235-46

[17] Sasidharan A, Swaroop S, Chandran P, Nair S and Koyakutty M 2016 Cellular and molecular mechanistic insight into the DNA-damaging potential of few-layer graphene in human primary endothelial cells Nanomedicine 12 1347-55

[18] Chowdhury S M, Fang J and Sitharaman B 2015 Interaction of graphene nanoribbons with components of the blood vascular system Future Sci. OA 1 FSO19

[19] Lei Z D, Wang J J, Wang L, Yang XY, Xu G and Tang L 2016 Efficient photocatalytic degradation of ibuprofen in aqueous solution using novel visible-light responsive graphene quantum $\operatorname{dot} / \mathrm{AgVO}_{3}$ nanoribbons J. Hazard. Mater. 312 298-306

[20] Talukdar Y, Rashkow J T, Lalwani G, Kanakia S and Sitharaman B 2014 The effects of graphene nanostructures on mesenchymal stem cells Biomaterials 35 4863-77

[21] Chng E L K and Pumera M 2015 Toxicity of graphene related materials and transition metal dichalcogenides RSC Adv. 5 3074-80

[22] Russier J, Treossi E, Scarsi A, Perrozzi F, Dumortier H, Ottaviano L, Meneghetti M, Palermo V and Bianco A 2013 Evidencing the mask effect of graphene oxide: a comparative study on primary human and murine phagocytic cells Nanoscale 5 11234-47

[23] Alberto B and Maurizio P 2015 Safety concerns on graphene and 2D materials: a flagship perspective 2D Mater. 2030201

[24] Liu S, Hu M, Zeng T H, Wu R, Jiang R, Wei J, Wang L, Kong J and Chen Y 2012 Lateral dimension-dependent antibacterial activity of graphene oxide sheets Langmuir 28 12364-72

[25] Perreault F, de Faria A F, Nejati S and Elimelech M 2015 Antimicrobial properties of graphene oxide nanosheets: why size matters ACS Nano 9 7226-36

[26] Ye S, Shao K, Li Z, Guo N, Zuo Y, Li Q, Lu Z, Chen L, He Q and Han H 2015 Antiviral activity of graphene oxide: how sharp edged structure and charge matter ACS Appl. Mater. Interfaces 721571-9

[27] Efremova L V, Vasilchenko A S, Rakov E G and Deryabin D G 2015 Toxicity of graphene shells, graphene oxide, and graphene oxide paper evaluated with Escherichia coli biotests Biomed. Res. Int. 2015869361

[28] Barbolina I, Woods C R, Lozano N, Kostarelos K, Novoselov K S and Roberts I S 2016 Purity of graphene oxide determines its antibacterial activity 2D Mater. 3025025

[29] Mukherjee S P, Lozano N, Kucki M, Del Rio-Castillo A E, Newman L, Vazquez E, Kostarelos K, Wick P and Fadeel B 2016 Detection of endotoxin contamination of graphene based materials using the TNF-alpha expression test and guidelines for endotoxin-free graphene oxide production PLoS One $11 \mathrm{e} 0166816$

[30] Combarros R G, Collado S and Diaz M 2016 Toxicity of graphene oxide on growth and metabolism of Pseudomonas putida J. Hazard. Mater. 310 246-52 
[31] Ge Y, Priester J H, Mortimer M, Chang C H, Ji Z, Schimel J P and Holden P A 2016 Long-term effects of multiwalled carbon nanotubes and graphene on microbial communities in dry soil Environ. Sci. Technol. 503965-74

[32] Zhang M, Yu Q, Liang C, Liu Z, Zhang B and Li M 2016 Graphene oxide induces plasma membrane damage, reactive oxygen species accumulation and fatty acid profiles change in Pichia pastoris Ecotoxicol. Environ. Saf. 132 372-8

[33] Hu C, Wang Q, Zhao H, Wang L, Guo S and Li X 2015 Ecotoxicological effects of graphene oxide on the protozoan Euglena gracilis Chemosphere 128 184-90

[34] Ouyang S, Hu X and Zhou Q 2015 Envelopmentinternalization synergistic effects and metabolic mechanisms of graphene oxide on single-cell chlorella vulgaris are dependent on the nanomaterial particle size ACS Appl. Mater. Interfaces 7 18104-12

[35] Nogueira P F, Nakabayashi D and Zucolotto V 2015 The effects of graphene oxide on green algae Raphidocelis subcapitata Aquat. Toxicol. 166 29-35

[36] Hu C, Hu N, Li X and Zhao Y 2016 Graphene oxide alleviates the ecotoxicity of copper on the freshwater microalga Scenedesmus obliquus Ecotoxicol. Environ. Saf. 132 360-5

[37] Gorka D E, Jeger J L, Zhang H, Ma Y, Colman B P, Bernhardt E S and Liu J 2016 Phytotoxicity of soluble graphitic nanofibers to model plant species Environ. Toxicol. Chem. 35 2941-7

[38] Liu S, Wei H, Li Z, Li S, Yan H, He Y and Tian Z 2015 Effects of graphene on germination and seedling morphology in rice J. Nanosci. Nanotechnol. 15 2695-701

[39] Hu X, Kang J, Lu K, Zhou R, Mu L and Zhou Q 2014 Graphene oxide amplifies the phytotoxicity of arsenic in wheat Sci. Rep. 46122

[40] Chatterjee N, Yang J S, Park K, Oh S M, Park J and Choi J 2015 Screening of toxic potential of graphene family nanomaterials using in vitro and alternative in vivo toxicity testing systems Environ. Health Toxicol. 30 e2015007

[41] Jung S K, Qu X, Aleman-Meza B, Wang T, Riepe C, Liu Z, Li Q and Zhong W 2015 Multi-endpoint, high-throughput study of nanomaterial toxicity in Caenorhabditis elegans Environ. Sci. Technol. 49 2477-85

[42] Wu Q, Zhou X, Han X, Zhuo Y, Zhu S, Zhao Y and Wang D 2016 Genome-wide identification and functional analysis of long noncoding RNAs involved in the response to graphene oxide Biomaterials 102 277-91

[43] Zhao Y, Wu Q and Wang D 2016 An epigenetic signal encoded protection mechanism is activated by graphene oxide to inhibit its induced reproductive toxicity in Caenorhabditis elegans Biomaterials 79 15-24

[44] Zhao Y, Yang R, Rui Q and Wang D 2016 Intestinal insulin signaling encodes two different molecular mechanisms for the shortened longevity induced by graphene oxide in Caenorhabditis elegans Sci. Rep. 624024

[45] Dziewiecka M, Karpeta-Kaczmarek J, Augustyniak M, Majchrzycki L and Augustyniak-Jablokow M A 2016 Evaluation of in vivo graphene oxide toxicity for Acheta domesticus in relation to nanomaterial purity and time passed from the exposure J. Hazard. Mater. 305 30-40

[46] Chen M, Yin J, Liang Y, Yuan S, Wang F, Song M and Wang H 2016 Oxidative stress and immunotoxicity induced by graphene oxide in zebrafish Aquat. Toxicol. 174 54-60

[47] Wang Z Get al 2015 Toxicity of graphene quantum dots in zebrafish embryo Biomed. Environ. Sci. 28 341-51

[48] Szmidt M, Sawosz E, Urbanska K, Jaworski S, Kutwin M, Hotowy A, Wierzbicki M, Grodzik M, Lipinska L and Chwalibog A 2016 Toxicity of different forms of graphene in a chicken embryo model Environ. Sci. Pollut. Res. Int. (doi: 10.1007/s11356-016-7178-z)

[49] Mottier A et al 2016 Surface area of carbon nanoparticles: a dose metric for a more realistic ecotoxicological assessment Nano Lett. 163514-8

[50] Ma J, Liu R, Wang X, Liu Q, Chen Y, Valle R P, Zuo Y Y, Xia T and Liu S 2015 Crucial role of lateral size for graphene oxide in activating macrophages and stimulating proinflammatory responses in cells and animals ACS Nano 9 10498-515
[51] Figarol A, Pourchez J, Boudard D, Forest V, Akono C, Tulliani J M, Lecompte J P, Cottier M, Bernache-Assollant D and Grosseau P 2015 In vitro toxicity of carbon nanotubes, nano-graphite and carbon black, similar impacts of acid functionalization Toxicol. In Vitro 30 476-85

[52] Wang X et al 2015 Use of a pro-fibrogenic mechanism-based predictive toxicological approach for tiered testing and decision analysis of carbonaceous nanomaterials ACS Nano 93032-43

[53] de Luna L A, de Moraes A C, Consonni S R, Pereira C D, Cadore S, Giorgio S and Alves O L 2016 Comparative in vitro toxicity of a graphene oxide-silver nanocomposite and the pristine counterparts toward macrophages J. Nanobiotechnol. 1412

[54] McIntyre J et al 2016 A comparison of catabolic pathways induced in primary macrophages by pristine single walled carbon nanotubes and pristine graphene RSCAdv. 6 65299-310

[55] Wu W, Yan L, Wu Q, Li Y, Li Q, Chen S, Yang Y, Gu Z, Xu H and Yin Z Q 2016 Evaluation of the toxicity of graphene oxide exposure to the eye Nanotoxicology 10 1329-40

[56] Liao K H, Lin Y S, Macosko C W and Haynes C L 2011 Cytotoxicity of graphene oxide and graphene in human erythrocytes and skin fibroblasts ACS Appl. Mater. Interfaces 32607-15

[57] Kucki M et al 2016 Interaction of graphene-related materials with human intestinal cells: an in vitro approach Nanoscale 8 8749-60

[58] Xu S, Xu S, Chen S, Fan H, Luo X, Yang X, Wang J, Yuan H, $\mathrm{Xu}$ A and Wu L 2016 Graphene oxide modulates B cell surface phenotype and impairs immunoglobulin secretion in plasma cell J. Nanosci. Nanotechnol. 16 4205-15

[59] Hashemi E, Akhavan O, Shamsara M, Daliri M, Dashtizad M and Farmany A 2016 Synthesis and cyto-genotoxicity evaluation of graphene on mice spermatogonial stem cells Colloids Surf. B 146 770-6

[60] Hu Q, Jiao B, Shi X, Valle R P, Zuo Y Y and Hu G 2015 Effects of graphene oxide nanosheets on the ultrastructure and biophysical properties of the pulmonary surfactant film Nanoscale 7 18025-9

[61] Liu X and Chen K L 2015 Interactions of graphene oxide with model cell membranes: probing nanoparticle attachment and lipid bilayer disruption Langmuir 31 12076-86

[62] Feng M, Kang H, Yang Z, Luan B and Zhou R 2016 Potential disruption of protein-protein interactions by graphene oxide J. Chem. Phys. 144225102

[63] Han S Get al 2015 Pulmonary responses of spraguedawley rats in single inhalation exposure to graphene oxide nanomaterials Biomed. Res. Int. 2015376756

[64] Kim J Ket al 201628 d inhalation toxicity of graphene nanoplatelets in Sprague-Dawley rats Nanotoxicology 10 891-901

[65] Lee J K, Jeong A Y, Bae J, Seok J H, Yang J Y, Roh H S, Jeong J, Han Y, Jeong J and Cho W S 2016 The role of surface functionalization on the pulmonary inflammogenicity and translocation into mediastinal lymph nodes of graphene nanoplatelets in rats Arch. Toxicol. (doi: 10.1007/s00204-0161706-y)

[66] Roberts J R et al 2016 Evaluation of pulmonary and systemic toxicity following lung exposure to graphite nanoplates: a member of the graphene-based nanomaterial family Part. Fibre Toxicol. 1334

[67] Mao L, Hu M, Pan B, Xie Y and Petersen E J 2016 Biodistribution and toxicity of radio-labeled few layer graphene in mice after intratracheal instillation Part. Fibre Toxicol. 137

[68] Wang K, Ruan J, Song H, Zhang J, Wo Y, Guo S and Cui D 2011 Biocompatibility of graphene oxide Nanoscale Res. Lett. 68

[69] Sasidharan A, Swaroop S, Koduri C K, Girish C M, Chandran P, Panchakarla L S, Somasundaram V H, Gowd G S, Nair S and Koyakutty M 2015 Comparative in vivo toxicity, organ biodistribution and immune response of pristine, carboxylated and PEGylated few-layer graphene sheets in Swiss albino mice: a three month study Carbon 95 511-24 
[70] Wen K P, Chen Y C, Chuang C H, Chang H Y, Lee C Y and Tai N H 2015 Accumulation and toxicity of intravenouslyinjected functionalized graphene oxide in mice J. Appl. Toxicol. 35 1211-8

[71] Mendonca M C, Soares E S, de Jesus M B, Ceragioli H J, Irazusta S P, Batista A G, Vinolo M A, Marostica Junior M R and da Cruz-Hofling M A 2016 Reduced graphene oxide: nanotoxicological profile in rats J. Nanobiotechnol. 1453

[72] Kanakia S, Toussaint J, Hoang D M, Mullick Chowdhury S, Lee S, Shroyer K R, Moore W, Wadghiri Y Z and Sitharaman B 2015 Towards an advanced graphene-based magnetic resonance imaging contrast agent: sub-acute toxicity and efficacy studies in small animals Sci. Rep. 517182

[73] Jasim D A, Menard-Moyon C, Begin D, Bianco A and Kostarelos K 2015 Tissue distribution and urinary excretion of intravenously administered chemically functionalized graphene oxide sheets Chem. Sci. 63952-64

[74] Kurantowicz N, Strojny B, Sawosz E, Jaworski S, Kutwin M, Grodzik M, Wierzbicki M, Lipinska L, Mitura K and Chwalibog A 2015 Biodistribution of a high dose of diamond, graphite, and graphene oxide nanoparticles after multiple intraperitoneal injections in rats Nanoscale Res. Lett. 10398

[75] Shang S, Yang S-Y, Liu Z-M and Yang X 2015 Oxidative damage in the kidney and brain of mice induced by different nano-materials Front. Biol. 10 91-6

[76] Patlolla A K, Randolph J, Kumari S A and Tchounwou P B 2016 Toxicity evaluation of graphene oxide in kidneys of Sprague-Dawley rats Int. J. Environ. Res. Public Health 13380

[77] Zhang D, Zhang Z, Liu Y, Chu M, Yang C, Li W, Shao Y, Yue Y and Xu R 2015 The short- and long-term effects of orally administered high-dose reduced graphene oxide nanosheets on mouse behaviors Biomaterials 68 100-13

[78] Xie Y, Wu B, Zhang XX, Yin J, Mao L and Hu M 2016 Influences of graphene on microbial community and antibiotic resistance genes in mouse gut as determined by high-throughput sequencing Chemosphere 144 1306-12

[79] Servant A, Leon V, Jasim D, Methven L, Limousin P, Fernandez-Pacheco E V, Prato M and Kostarelos K 2014 Graphene-based electroresponsive scaffolds as polymeric implants for on-demand drug delivery Adv. Healthcare Mater. $31334-43$

[80] Yang T, Wang W, Zhang H, Li X, Shi J, He Y, Zheng Q S, Li Z and Zhu H 2015 Tactile sensing system based on arrays of graphene woven microfabrics: electromechanical behavior and electronic skin application ACS Nano 9 10867-75

[81] Kuang J, Dai Z, Liu L, Yang Z, Jin M and Zhang Z 2015 Synergistic effects from graphene and carbon nanotubes endow ordered hierarchical structure foams with a combination of compressibility, super-elasticity and stability and potential application as pressure sensors Nanoscale 7 9252-60

[82] Tian H, Shu Y, Cui Y L, Mi W T, Yang Y, Xie D and Ren T L 2014 Scalable fabrication of high-performance and flexible graphene strain sensors Nanoscale 6 699-705

[83] Zhu B, Niu Z, Wang H, Leow W R, Wang H, Li Y, Zheng L, Wei J, Huo F and Chen X 2014 Microstructured graphene arrays for highly sensitive flexible tactile sensors Small 10 3625-31

[84] Sun J, Deng Y, Li J, Wang G, He P, Tian S, Bu X, Di Z, Yang S, Ding G and Xie X 2016 A new graphene derivative: hydroxylated graphene with excellent biocompatibility ACS Appl. Mater. Interfaces 8 10226-33

[85] Ema M, Matsuda A, Kobayashi N, Naya M and Nakanishi J 2011 Evaluation of dermal and eye irritation and skin sensitization due to carbon nanotubes Regul. Toxicol. Pharmacol. 61 276-81

[86] Robertson T A, Sanchez W Y and Roberts M S 2010 Are commercially available nanoparticles safe when applied to the skin? J. Biomed. Nanotechnol. 6452-68

[87] Shvedova A A, Castranova V, Kisin E R, Schwegler-Berry D, Murray A R, Gandelsman V Z, Maynard A and Baron P 2003 Exposure to carbon nanotube material: assessment of nanotube cytotoxicity using human keratinocyte cells J. Toxicol. Environ. Health A 66 1909-26

[88] Zhao J, Deng B, Lv M, Li J, Zhang Y, Jiang H, Peng C, Li J, Shi J, Huang Q and Fan C 2013 Graphene oxide-based antibacterial cotton fabrics Adv. Healthcare Mater. 2 1259-66
[89] Diez-Pascual A M and Diez-Vicente A L 2016 Poly(propylene fumarate)/polyethylene glycol-modified graphene oxide nanocomposites for tissue engineering ACS Appl. Mater. Interfaces 8 17902-14

[90] Cedervall T, Lynch I, Lindman S, Berggard T, Thulin E, Nilsson H, Dawson K A and Linse S 2007 Understanding the nanoparticle-protein corona using methods to quantify exchange rates and affinities of proteins for nanoparticles Proc. Natl Acad. Sci. USA 1042050-5

[91] Lynch I, Cedervall T, Lundqvist M, Cabaleiro-Lago C, Linse S and Dawson KA 2007 The nanoparticle-protein complex as a biological entity; a complex fluids and surface science challenge for the 21st century Adv. Colloid Interface Sci. 134-5 167-74

[92] Lundqvist M, Stigler J, Elia G, Lynch I, Cedervall T and Dawson K A 2008 Nanoparticle size and surface properties determine the protein corona with possible implications for biological impacts Proc. Natl Acad. Sci. USA 105 14265-70

[93] Volkov Y2015 Quantum dots in nanomedicine: recent trends, advances and unresolved issues Biochem. Biophys. Res. Commun. 468 419-27

[94] Nel A E, Madler L, Velegol D, Xia T, Hoek E M, Somasundaran P, Klaessig F, Castranova V and Thompson M 2009 Understanding biophysicochemical interactions at the nano-bio interface Nat. Mater. 8543-57

[95] Xu M, Zhu J, Wang F, Xiong Y, Wu Y, Wang Q, Weng J, Zhang Z, Chen W and Liu S 2016 Improved in vitro and in vivo biocompatibility of graphene oxide through surface modification: poly(acrylic acid)-functionalization is superior to PEGylation ACS Nano $103267-81$

[96] Duan G, Kang S G, Tian X, Garate J A, Zhao L, Ge C and Zhou R 2015 Protein corona mitigates the cytotoxicity of graphene oxide by reducing its physical interaction with cell membrane Nanoscale 7 15214-24

[97] Chong Y, Ge C, Yang Z, Garate J A, Gu Z, Weber J K, Liu J and Zhou R 2015 Reduced cytotoxicity of graphene nanosheets mediated by blood-protein coating ACS Nano 9 5713-24

[98] Mu L, Gao Y and Hu X 2016 Characterization of biological secretions binding to graphene oxide in water and the specific toxicological mechanisms Environ. Sci. Technol. 50 8530-7

[99] Kagan VEet al 2010 Carbon nanotubes degraded by neutrophil myeloperoxidase induce less pulmonary inflammation Nat. Nanotechnol. 5354-9

[100] Bhattacharya K, Mukherjee S P, Gallud A, Burkert S C, Bistarelli S, Bellucci S, Bottini M, Star A and Fadeel B 2016 Biological interactions of carbon-based nanomaterials: from coronation to degradation Nanomedicine 12 333-51

[101] Sasaki K-i, Tokura Y and Sogawa T 2013 The origin of raman $\mathrm{d}$ band: bonding and antibonding orbitals in graphene Crystals 3120

[102] Liu Y et al 2016 Graphene oxide attenuates the cytotoxicity and mutagenicity of PCB 52 via activation of genuine autophagy Environ. Sci. Technol. 50 3154-64

[103] Vlasova I I, Kapralov A A, Michael Z P, Burkert S C, Shurin M R, Star A, Shvedova A A and Kagan V E 2016 Enzymatic oxidative biodegradation of nanoparticles: mechanisms, significance and applications Toxicol. Appl. Pharmacol. 299 58-69

[104] Zhang W, Yan L, Li M, Zhao R, Yang X, Ji T, Gu Z, Yin JJ, Gao X and Nie G 2015 Deciphering the underlying mechanisms of oxidation-state dependent cytotoxicity of graphene oxide on mammalian cells Toxicol. Lett. 237 61-71

[105] Wang X Y, Lei R, Huang H D, Wang N, Yuan L, Xiao R Y, Bai L D, Li X, Li L M and Yang X D 2015 The permeability and transport mechanism of graphene quantum dots (GQDs) across the biological barrier Nanoscale 7 2034-41

[106] Choi H S, Liu W, Misra P, Tanaka E, Zimmer J P, Ipe B I, Bawendi M G and Frangioni JV 2007 Renal clearance of quantum dots Nat. Biotechnol. 25 1165-70

[107] Jasim D A, Boutin H, Fairclough M, Ménard-Moyon C, Prenant C, Bianco A and Kostarelos K 2016 Thickness of functionalized graphene oxide sheets plays critical role in tissue accumulation and urinary excretion: a pilot PET/CT study Appl. Mater. Today 424-30 
[108] Guan M R et al 2016 Fullerene/photosensitizer nanovesicles as highly efficient and clearable phototheranostics with enhanced tumor accumulation for cancer therapy Biomaterials $10375-85$

[109] Guan M R, Li J, Jia Q Y, Ge J C, Chen D Q, Zhou Y, Wang P F, Zou T J, Zhen M M, Wang C R and Shu C Y 2016 A versatile and clearable nanocarbon theranostic based on carbon dots and gadolinium metallofullerene nanocrystals Adv. Healthcare Mater. 5 2283-94

[110] Parvin N and Mandal T K 2016 Synthesis of a highly fluorescence nitrogen-doped carbon quantum dots bioimaging probe and its in vivo clearance and printing applications RSCAdv. $618134-40$

[111] OECD 2007-2016 Testing programme of manufactured nanomaterials www.oecd.org/chemicalsafety/nanosafety/ overview-testing-programme-manufactured-nanomaterials htm (Accessed: 01/09/2016)

[112] Movia D et al 2014 A safe-by-design approach to the development of gold nanoboxes as carriers for internalization into cancer cells Biomaterials 35 2543-57

[113] Dekkers S et al 2016 Towards a nanospecific approach for risk assessment Regul. Toxicol. Pharmacol. 80 46-59

[114] De Marzi L, Ottaviano L, Perrozzi F, Nardone M, Santucci S, De Lapuente J, Borras M, Treossi E, Palermo V and Poma A 2014 Flake size-dependent cyto and genotoxic evaluation of graphene oxide on in vitro $\mathrm{A} 549, \mathrm{CaCo}_{2}$ and vero cell lines J. Biol. Regul. Homeost. Agents 28 281-9

[115] Jiang Y, Raliya R, Fortner J D and Biswas P 2016 Graphene oxides in water: correlating morphology and surface chemistry with aggregation behavior Environ. Sci. Technol. 50 6964-73

[116] Suzuki N et al 2016 Chiral graphene quantum dots ACS Nano $101744-55$

[117] Karahan H E, Wei L, Goh K, Wiraja C, Liu Z, Xu C, Jiang R, Wei J and Chen Y 2016 Synergism of water shock and a biocompatible block copolymer potentiates the antibacterial activity of graphene oxide Small 12 951-62

[118] Pinto A M, Moreira J A, Magalhaes F D and Goncalves I C 2016 Polymer surface adsorption as a strategy to improve the biocompatibility of graphene nanoplatelets Colloids Surf. B 146818-24

[119] Singh S K, Singh M K, Kulkarni P P, Sonkar V K, Gracio J J and Dash D 2012 Amine-modified graphene: thromboprotective safer alternative to graphene oxide for biomedical applications ACS Nano 6 2731-40

[120] Zhang X, Sui M, Yan X, Huang T and Yuan Z 2016 Mitigation in the toxicity of graphene oxide nanosheets towards Escherichia coli in the presence of humic acid Environ. Sci. Process Impacts 18 744-50

[121] Zhao Y, Yu X, Jia R, Yang R, Rui Q and Wang D 2015 Lactic acid bacteria protects Caenorhabditis elegans from toxicity of graphene oxide by maintaining normal intestinal permeability under different genetic backgrounds Sci. Rep. 517233

[122] Gurunathan S, Han J W, Park J H, Eppakayala V and Kim J H 2014 Ginkgo biloba: a natural reducing agent for the synthesis of cytocompatible graphene Int. J. Nanomed. 9363-77

[123] Kim H, Kim J, Lee M, Choi H C and Kim W J 2016 Stimuliregulated enzymatically degradable smart grapheneoxide-polymer nanocarrier facilitating photothermal gene delivery Adv. Healthcare Mater. 5 1918-30

[124] Zan P, Yang C, Sun H, Zhao L, Lv Z and He Y 2016 One-pot fabricating $\mathrm{Fe}_{3} \mathrm{O}_{4}$ /graphene nanocomposite with excellent biocompatibility and non-toxicity as a negative MR contrast agent Colloids Surf. B 145 208-16

[125] de Moraes A C, Lima B A, de Faria A F, Brocchi M and Alves O L 2015 Graphene oxide-silver nanocomposite as a promising biocidal agent against methicillin-resistant Staphylococcus aureus Int. J. Nanomed. 10 6847-61

[126] Jankauskaite V, Vitkauskiene A, Lazauskas A, Baltrusaitis J, Prosycevas I and Andrulevicius M 2016 Bactericidal effect of graphene oxide/Cu/Ag nanoderivatives against Escherichia coli, Pseudomonas aeruginosa, Klebsiella pneumoniae, Staphylococcus aureus and methicillin-resistant Staphylococcus aureus Int. J. Pharm. 511 90-7
[127] Buccheri M A et al 2016 Modification of graphene oxide by laser irradiation: a new route to enhance antibacterial activity Nanotechnology 27245704

[128] Fang J, Nakamura H and Maeda H 2011 The EPR effect: unique features of tumor blood vessels for drug delivery, factors involved, and limitations and augmentation of the effect Adv. Drug Deliv. Rev. 63 136-51

[129] Maeda H, Wu J, Sawa T, Matsumura Y and Hori K 2000 Tumor vascular permeability and the EPR effect in macromolecular therapeutics: a review J. Control. Release $65271-84$

[130] Choi Y J, Kim E, Han J, Kim J H and Gurunathan S 2016 A novel biomolecule-mediated reduction of graphene oxide: a multifunctional anti-cancer agent Molecules 21375

[131] Park C S, Choi K S, Shin J W and Kim S Y 2015 Inhibition of viability of the respiratory epithelial cells using functionalized graphene oxide J. Nanosci. Nanotechnol. 15 2060-6

[132] Khatamian M, Divband B and Farahmand-Zahed F 2016 Synthesis and characterization of zinc (II)-loaded zeolite/ graphene oxide nanocomposite as a new drug carrier Mater. Sci. Eng. C 66 251-8

[133] Wo F, Xu R, Shao Y, Zhang Z, Chu M, Shi D and Liu S 2016 A multimodal system with synergistic effects of magnetomechanical, photothermal, photodynamic and chemo therapies of cancer in graphene-quantum dot-coated hollow magnetic nanospheres Theranostics 6 485-500

[134] Xu L, Xiang J, Liu Y, Xu J, Luo Y, Feng L, Liu Z and Peng R 2016 Functionalized graphene oxide serves as a novel vaccine nano-adjuvant for robust stimulation of cellular immunity Nanoscale 83785-95

[135] Pieper H, Chercheja S, Eigler S, Halbig C E, Filipovic M R and Mokhir A 2016 Endoperoxides revealed as origin of the toxicity of graphene oxide Angew. Chem., Int. Ed. Engl. 55 405-7

[136] Fiorillo M, Verre A F, Iliut M, Peiris-Pages M, Ozsvari B, Gandara R, Cappello A R, Sotgia F, Vijayaraghavan A and Lisanti M P 2015 Graphene oxide selectively targets cancer stem cells, across multiple tumor types: implications for nontoxic cancer treatment, via 'differentiation-based nano-therapy' Oncotarget 6 3553-62

[137] Hu D, Zhang J, Gao G, Sheng Z, Cui H and Cai L 2016 Indocyanine green-loaded polydopamine-reduced graphene oxide nanocomposites with amplifying photoacoustic and photothermal effects for cancer theranostics Theranostics $61043-52$

[138] Chen J, Liu C, Zeng G, You Y, Wang H, Gong X, Zheng R, Kim J, Kim C and Song L 2016 Indocyanine green loaded reduced graphene oxide for in vivo photoacoustic/fluorescence dualmodality tumor imaging Nanoscale Res. Lett. 1185

[139] Luo S, Yang Z, Tan X, Wang Y, Zeng Y, Wang Y, Li C, Li R and Shi C 2016 Multifunctional photosensitizer grafted on polyethylene glycol and polyethylenimine dualfunctionalized nanographene oxide for cancer-targeted nearinfrared imaging and synergistic phototherapy ACS Appl. Mater. Interfaces 8 17176-86

[140] Yang D et al 2016 In vivo targeting of metastatic breast cancer via tumor vasculature-specific nano-graphene oxide Biomaterials 104361-71

[141] Zhang X, Yang C, Zhou J and Huo M 2016 Somatostatin receptor-mediated tumor-targeting nanocarriers based on octreotide-PEG conjugated nanographene oxide for combined chemo and photothermal therapy Small 12 3578-90

[142] Zhou B, Huang Y, Yang F, Zheng W and Chen T 2016 Dualfunctional nanographene oxide as cancer-targeted drugdelivery system to selectively induce cancer-cell apoptosis Chem. Asian J. 11 1008-19

[143] Hou L, Shi Y, Jiang G, Liu W, Han H, Feng Q, Ren J, Yuan Y, Wang Y, Shi J and Zhang Z 2016 Smart nanocomposite hydrogels based on azo crosslinked graphene oxide for oral colon-specific drug delivery Nanotechnology 27315105

[144] Upadhyay R, Naskar S, Bhaskar N, Bose S and Basu B 2016 Modulation of protein adsorption and cell proliferation on 
polyethylene immobilized graphene oxide reinforced HDPE bionanocomposites ACS Appl. Mater. Interfaces 8 11954-68

[145] Garcia-Alegria E, Iluit M, Stefanska M, Silva C, Heeg S, Kimber S J, Kouskoff V, Lacaud G, Vijayaraghavan A and Batta K 2016 Graphene oxide promotes embryonic stem cell differentiation to haematopoietic lineage Sci. Rep. 625917

[146] Kumar S and Chatterjee K 2016 Comprehensive review on the use of graphene-based substrates for regenerative medicine and biomedical devices ACS Appl. Mater. Interfaces 826431-57

[147] Shin S R, Li Y C, Jang H L, Khoshakhlagh P, Akbari M, Nasajpour A, Zhang Y S, Tamayol A and Khademhosseini A 2016 Graphene-based materials for tissue engineering Adv. Drug Deliv. Rev. 105 255-74

[148] Zhou K, Motamed S, Thouas G A, Bernard C C, Li D, Parkington H C, Coleman H A, Finkelstein D I and
Forsythe J S 2016 Graphene functionalized scaffolds reduce the inflammatory response and supports endogenous neuroblast migration when implanted in the adult brain PLoS One 11 e0151589

[149] Kulshrestha S, Khan S, Meena R, Singh B R and Khan A U 2014 A graphene/zinc oxide nanocomposite film protects dental implant surfaces against cariogenic Streptococcus mutans Biofouling 30 1281-94

[150] La W G, Jin M, Park S, Yoon H H, Jeong G J, Bhang S H, Park H, Char K and Kim B S 2014 Delivery of bone morphogenetic protein- 2 and substance $\mathrm{P}$ using graphene oxide for bone regeneration Int. J. Nanomed. 9 107-16

[151] Li J, Wang G, Geng H, Zhu H, Zhang M, Di Z, Liu X, Chu P K and Wang X 2015 CVD growth of graphene on NiTi alloy for enhanced biological activity ACS Appl. Mater. Interfaces 7 19876-81 